\title{
Review \\ Quantifying the Benefits and Ecosystem Services Provided by Green Roofs-A Review
}

\author{
Cuong Ngoc Nguyen ${ }^{1}\left(\mathbb{D}\right.$, Nitin Muttil ${ }^{1,2, *(\mathbb{D}}$, Muhammad Atiq Ur Rehman Tariq ${ }^{1,2}$ and Anne W. M. Ng ${ }^{3, *(\mathbb{C})}$ \\ 1 Institute for Sustainable Industries \& Liveable Cities, Victoria University, Melbourne, VIC 8001, Australia; \\ ngoc.nguyen178@live.vu.edu.au (C.N.N.); atiq.tariq@yahoo.com (M.A.U.R.T.) \\ 2 College of Engineering and Science, Victoria University, Melbourne, VIC 8001, Australia \\ 3 College of Engineering, Information Technology and Environment, Charles Darwin University, \\ Ellengowan Dr, Brinkin, NT 0810, Australia \\ * Correspondence: nitin.muttil@vu.edu.au (N.M.); anne.ng@cdu.edu.au (A.W.M.N.)
}

check for updates

Citation: Nguyen, C.N.; Muttil, N.; Tariq, M.A.U.R.; Ng, A.W.M. Quantifying the Benefits and Ecosystem Services Provided by Green Roofs-A Review. Water 2022, 14, 68. https://doi.org/10.3390/ w14010068

Academic Editor: Michael Richter

Received: 28 November 2021

Accepted: 23 December 2021

Published: 31 December 2021

Publisher's Note: MDPI stays neutral with regard to jurisdictional claims in published maps and institutional affiliations.

Copyright: (c) 2021 by the authors. Licensee MDPI, Basel, Switzerland. This article is an open access article distributed under the terms and conditions of the Creative Commons Attribution (CC BY) license (https:// creativecommons.org/licenses/by/ $4.0 /)$.

\begin{abstract}
Water-sensitive urban design (WSUD) has been widely used in cities to mitigate the negative consequences of urbanization and climate change. One of the WSUD strategies that is becoming popular is green roofs (GR) which offer a wide range of ecosystem services. Research on this WSUD strategy has been continuously increasing in terms of both quantity and quality. This paper presents a comprehensive review quantifying the benefits of GRs in papers published since 2010. More precisely, this review aims to provide up-to-date information about each GR benefit and how they have improved over the last decade. In agreement with previous reviews, extensive GRs were considerably researched, as compared to very limited studies on intensive and semi-intensive GRs. Each GR ecosystem service was specifically quantified, and an imbalance of GR research focus was identified, wherein urban heat- and runoff-related benefits were outstandingly popular when compared to other benefits. The results also highlight the recent introduction of hybrid GRs, which demonstrated improvements in GR performance. Furthermore, limitations of GRs, obstacles to their uptake, and inconsistent research findings were also identified in this review. Accordingly, opportunities for future research were pointed out in this review. This paper also recommends future studies to improve upon well-known GR benefits by exploring and applying more innovative GR construction techniques and materials. At the same time, further studies need to be undertaken on inadequately studied GR benefits, such as reduced noise and air pollution. In spite of the existence of reliable modelling tools, their application to study the effects of large-scale implementations of GRs has been restricted. Insufficient information from such research is likely to restrict large-scale implementations of GRs. As a result, further studies are required to transform the GR concept into one of the widely accepted and implemented WSUD strategies.
\end{abstract}

Keywords: WSUD; green roofs; ecosystem services; quantify benefits; large-scale implementation

\section{Introduction}

Water-sensitive urban design (WSUD) strategies have been widely used in cities to mitigate the negative consequences of rapid urbanization and climate change. Whilst WSUD is a popular term in Australia and a few other countries, blue-green infrastructure (BGI) is also a commonly used term. Green roofs (GR) have been regarded as a promising BGI strategy to deal with these globally growing concerns of urbanization and climate change. This BGI strategy, sometimes called a living roof or vegetated roof, offers a wide range of environmental, social, and economic benefits compared to conventional roofs (CRs) [1]. The stormwater management and the mitigation of heat-related issues are the two primary GR benefits that have attracted the attention of researchers the most. Additionally, GR is capable of reducing energy consumption, improving air and runoff quality, and alleviating noise pollution [2]. The vegetated roof also enhances the aesthetic aspects of a building and urban ecology by converting impervious roof surfaces to green spaces [3]. 
The configurations of a GR vary according to its geographical location, requirements, and the purposes for which it is built. Generally, a typical GR consists of the following layers (from the bottom to the top): waterproofing membrane, drainage layer, filter layer, substrate (growing medium), and vegetation layer. The insulation layer is optional and added when GRs are implemented on existing roofs (i.e., retrofitting a green roof). In the event that long-rooted plants are applied, anti-root layers are compulsory to protect both the GR system and its underneath structure [1]. In terms of GR types, GRs are categorized into intensive GRs (IGRs) and extensive GRs (EGRs). The main difference between these two groups is the substrate depth. While the IGR substrate is more than $20 \mathrm{~cm}$ thick, the EGR growing medium is thinner, with less than $15 \mathrm{~cm}$ [4]. Consequently, IGRs are suitable for the vast majority of plants, whereas EGRs are only able to support the survival of drought-resilient plants, such as succulents. By contrast, EGRs are much more prevalent than IGRs for several reasons, including lesser efforts for maintenance, lighter weights, and lower construction costs [5,6]. A semi-intensive GR (SIGR) is a type of GR with an intermediate substrate depth between those of EGRs and IGRs. A moderate substrate thickness allows a SIGR to accommodate small shrubs [7].

Though the success of GR projects at a pilot- (or small-) scale as well as at a large-scale have been reported globally, there is still a long way to go towards global acceptance and implementation of this innovative type of roof. This could be a result of the unbalanced focus on GRs between developed countries and the rest of the world. The reviewed papers are distributed across various countries, as presented in Figure 1. Figure 1 points out that most of the 102 papers reviewed in this study are from the USA, Europe, and other developed countries. The last decade saw the implementation of GRs in other countries such as Australia, China, Hong Kong, and Italy. The USA is still leading in terms of GR research, which was also reported by other studies, such as Blank et al. [8]. A more recent study by Zheng et al. [9] has also provided a similar finding. They studied 75 papers analyzing runoff retention in GRs and found that minimal efforts have been made to study and implement GRs in some regions of the world, including Africa, Central America, and Central Asia. This lack of GR-related research and knowledge in developing or underdeveloped countries leads to stakeholders, such as building owners, developers, and builders, being unaware of the benefits and optimal components of GRs appropriate for their study locations and roof sites $[1,2]$.

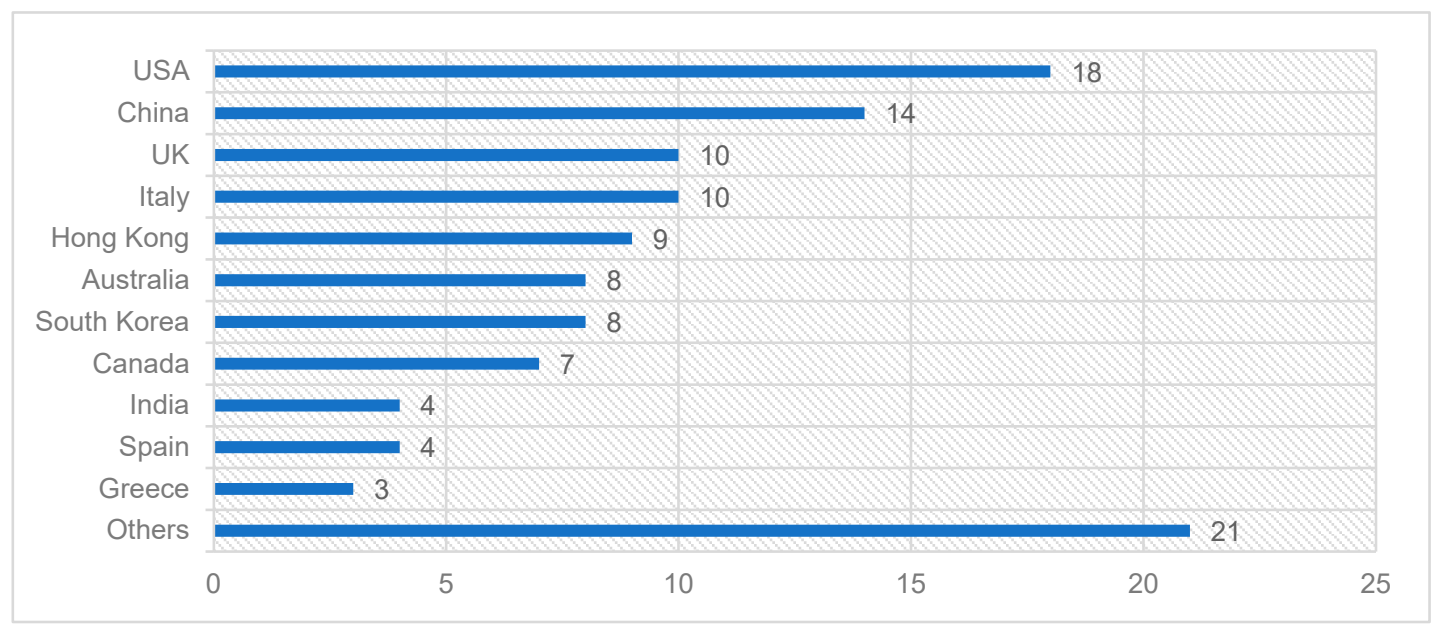

Figure 1. Distribution of reviewed papers by their country of implementation.

As far as the scale of GR implementation is concerned, they have been implemented at various spatial scales. In this study, they are grouped into three categories: pilot-scale (or small-scale), full-scale, and large-scale. Pilot-scale GRs occupy a small portion of the roof area and/or they are commonly installed on raised test beds or modules, whereas full-scale GRs are those covering the entire roof area with some non-vegetated paths for 
rooftop access, maintenance, and use of equipment [10]. Finally, the term "large-scale" refers to studies considering the application of GRs at scales that exceed the single-building scale, such as the city-wide scale, municipal scale, or catchment scale. Figure 2 indicates the domination of pilot-scale and full-scale GR studies, whereas only 10 studies (i.e., 10\% of the reviewed studies) investigated the potential of implementing GRs at a large scale. The large-scale implementation of GRs is constrained by several factors. For instance, the installation and maintenance costs for a GR remain high, which requires further studies to explore GR components that are not just economical but are also environmentally friendly. Another barrier could be the knowledge gap in quantifying the benefits of the large-scale implementation of GRs. There are 12 studies which did not conduct a field experiment and/or did not investigate large-scale GRs through simulation models. They were, hence, categorized as "others". Though the prospects of GRs at large scales are reasonably foreseeable, the insufficient information from limited research is likely to prevent authorities from issuing policies that encourage the large-scale implementation of GRs (such as financial incentives and utility bill reduction) [2].

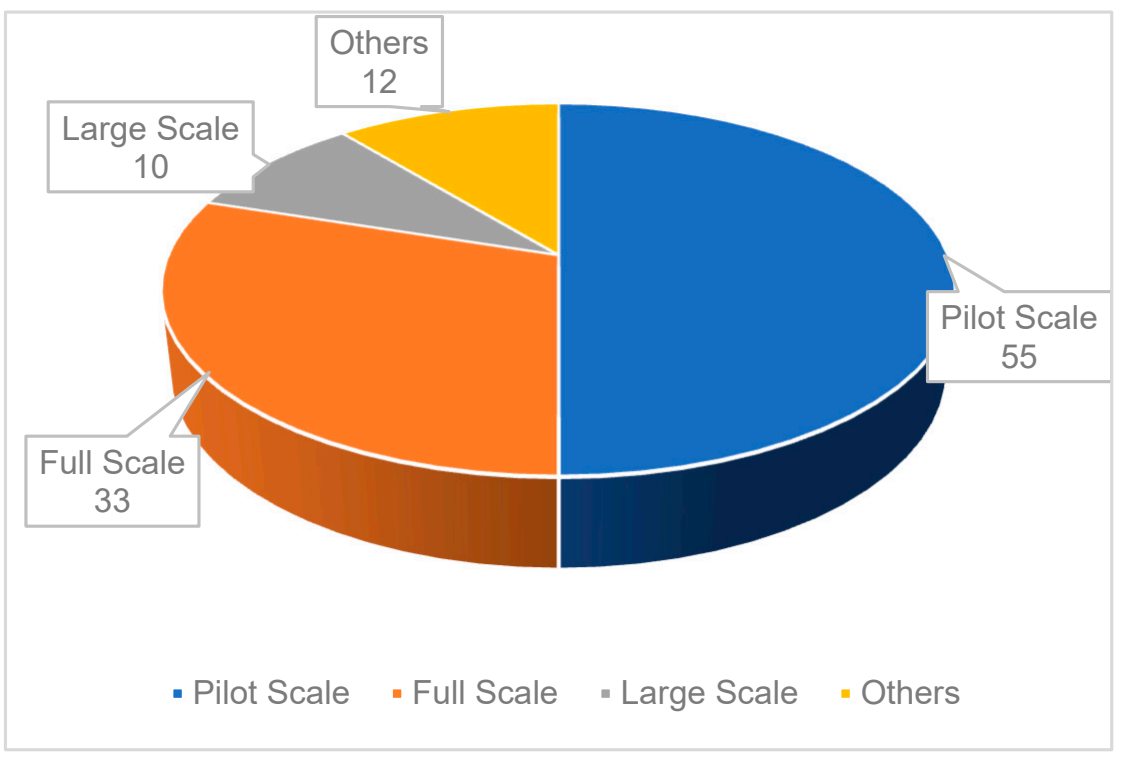

Figure 2. Break-down of papers based on the GR scale.

Earlier literature reviews on GRs concentrated on exploring their potential benefits, components, challenges, trends, and implementation opportunities [1,2,11,12]. Other previous works studied the performance of GRs implemented in different locations and climatic characteristics $[4,5,13-15]$. This review has also identified that the majority of the research conducted was qualitative based. Therefore, there is a need to quantify the benefits of GRs in order to demonstrate the need of more GR research areas to be investigated. This paper aims to conduct a comprehensive review to quantify GR benefits based on papers published during the last decade (from 2010 onwards) by considering several functional indicators, such as surface and air temperature (thermal reduction) and rainfall retention and peak flow (runoff reduction). Furthermore, this paper provides up-to-date information on each of the GR benefits that have been studied and whose performance has been improved during the last decade.

This paper is structured as follows. An overview of the reviewed papers and a summary of different types of GRs is presented in the next section. This is followed by the quantification of various GR benefits. The paper is concluded with a discussion of the key outcomes of this review and, finally, conclusions are drawn and recommendations from this study are presented. 


\section{Overview of Literature}

\subsection{Methodology}

This review of GR papers used the Google Scholar and Scopus search engines as the primary search database with a timeframe ranging from 2010 to 2021 . Within a set of potential search keywords for GRs, only "green roof" was chosen. Compared to other possible keywords such as "vegetated roof", "cool roof", and "living roof", the selected keyword was able to cover all potential papers and thus satisfy the study objectives. In particular, this selection aims to draw conclusions about how significant each of the GR benefits has been in studies to date. Most relevant papers were identified through an exhaustive abstract-screening procedure. This method enables the filtering process to exclude scholarly works which are not associated with the scope of the present study, such as review papers and papers not focusing on the quantification of GR performance. The search process aimed to identify at least 100 suitable papers. The authors of this review are aware that there are substantially more works and published materials on GRs during the selected time frame. The papers studied in this review were selected based on their relevance in terms of quantifying the benefits of GRs. The work reviewed in this study provides a good snapshot of current research on GRs and ensures that sufficient information is available for the analysis and that a good balance of the number of papers is available from each year, from 2010 onwards.

The information of papers identified in the search process described above will be divided into several categories to facilitate the quantifying process. Firstly, the preliminary categorization is presented in Table A1 (in Appendix A), which provides the reader with an overview of the GR-related research with respect to the location, country, type of GR, methodology used, and GR benefits investigated. Later, this review goes into an in-depth evaluation of GR benefits and attempts to quantify them.

\subsection{Types of Green Roof}

\subsubsection{Traditional Green Roofs}

As discussed above, GRs are traditionally divided into three groups: EGR, SIGR, and IGR. The main difference between them is the depth of the substrate layer and the corresponding plants to suit a particular depth. EGRs with numerous favorable characteristics are widely implemented. Figure 3 describes the distribution of papers across the different GR types. It is worth highlighting that EGRs have been extensively studied, with 80 out of the 102 reviewed papers being on EGRs. The strong preference for EGRs has been well documented in previous studies and remains unchanged to date. As already mentioned, EGRs have been thoroughly and widely implemented because of several advantages, such as ease of installation due to their light weight, low initial and operational costs, and less maintenance requirements [16]. A good example of a country where EGRs are highly popular is Germany, where $85 \%$ of new GRs constructed annually are EGRs [17]. He et al. [18] studied the changes in temperature and energy consumption in test chambers with extensive green modules on their top surface. Ávila-Hernández et al. [19] collected the experimental data from an EGR on a test box to validate the simulation results of EnergyPlus software. Liu et al. [20] used EGR-based parameters as the inputs of the Storm Water Management Model (SWMM) to assess the GR's capability of mitigating urban flooding for Nanchang in China. Cascone et al. [21] simulated a variety of EGRs through EnergyPlus to comprehensively study the performance of a GR on a building in Catania, in Sicily, Italy. Palermo et al. [22] investigated the hydrological behaviour of a full-scale EGR on top of a building at the University of Calabria, Italy. Carson, Marasco, Culligan and McGillis [10] monitored the retention capacity of three full-scale EGRs for one year and successively developed a model to identify the multi-year hydrological response of these systems. 




Figure 3. Distribution of papers across each type of GR (EGR: extensive green roof, IGR: intensive green roof, and SIGR: semi-intensive green roof).

On the other hand, SIGRs and IGRs have received less attention from researchers, although they outperform EGRs with regard to ecosystem services, mainly due to their thicker substrate layer. This lack of attention could be because of the stronger structural requirements, higher costs, and frequent maintenance associated with SIGRs. Of the 102 reviewed papers, a limited number of studies have considered SIGRs and IGRs, with only 11 and 15 studies being related to SIGRs and IGRs, respectively. For example, Lee and Jim [23] studied a full-scale IGR with a very deep substrate of $1 \mathrm{~m}$ to observe its thermal and cooling performance in Hong Kong. Such a thick substrate can allow the growth and survival of woodland vegetation. Kratschmer et al. [24] examined the impact of various full-scale GRs with thick substrates (up to $0.9 \mathrm{~m}$ ) on the diversity and abundance of wild bees. Beecham and Razzaghmanesh [25] investigated both the water quantity and quality of several pilot-scale EGRs and IGRs in Adelaide, Australia, with $0.1 \mathrm{~m}$ and $0.3 \mathrm{~m}$ of substrate depth, respectively. Moreover, researchers have not input the configurations of SIGRs and IGRs into their simulation models due to the low possibility of the application of these GRs. There are some exceptions to this. Morakinyo et al. [26] used ENVI-met and EnergyPlus to simulate and evaluate the reduction of temperature and the energy use of a building installing an IGR system. Baek et al. [27] collected the field data of a pilot-scale SIGR for inputs of the coupled SWMM and HYDRUS model to estimate the runoff reduction throughout an urban sub-basin.

\subsubsection{Hybrid Green Roofs}

In a broader context, researchers have recently attempted to improve the ecosystem services of this WSUD practice by integrating GRs with other systems. In this review, the term "Hybrid GR" is used to represent such roofs. One of them is the green-blue roof initially developed in Korea [1]. This is a combination of a blue roof and a green roof. A green-blue roof is technically similar to a typical GR, with the addition of one storage layer below the growing medium (Figure 4a). Only three papers (from the 102 reviewed papers) studied this type of roof. These are the studies by Shafique et al. [28], Shafique et al. [29], and Shafique and Kim [30]. This modified GR was reported to significantly mitigate urban flooding and urban heat island phenomena. Additionally, the integration of photovoltaic (PV) modules with GRs is a globally increasing trend among researchers. The integration 
of these two systems brings benefits in terms of both the electrical production and GR services [31]. More precisely, the evapotranspiration process of GR plants and growing mediums lessens the surface temperature of PV panels as well as their surrounding environment, which improves the electrical yield [32]. PV panels, which partially shade the surface of the GR, enhance GR benefits by limiting the solar radiation and, hence, diminishing the evapotranspiration rate of GRs [2].

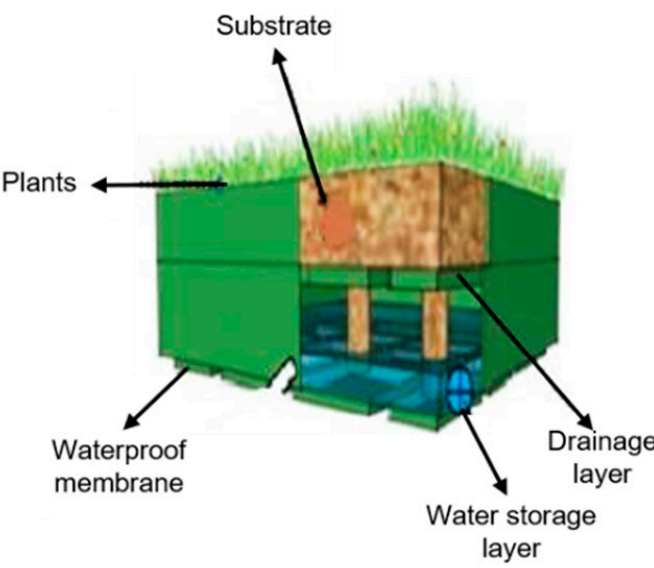

(a)

Figure 4. Schematic diagram of (a) a typical green-blue roof and (b) a pilot-scale green roof integrated with a radiant cooling system.

The other two types of hybrid GRs identified in this review are the GR-green wall system and the integration of GRs with radiant cooling systems. The construction of a vertical greening system along with a living roof is expected to greatly improve the Human Thermal Comfort (HTC) and thus reduce the cooling and heating demand. Only three papers (out of the 102 reviewed papers) considered this type of hybrid GR. Because of the difficulties and challenges associated with carrying out such projects at a building scale, previous studies explored the thermal behaviour of this combination in an experimental room [33] and with pilot-scale prototypes simulating full-scale dwellings [34,35]. In addition, the present study also found two papers examining the performance of a radiant cooling system integrated with a GR (Figure 4b). This type of hybrid GR comprises of a water pipe system (radiant component) and a sprinkler system (evaporative component) in its configuration [36]. It was determined that this hybrid GR performed better than conventional GRs in terms of temperature and energy reduction [36,37].

\section{Quantification of Green Roof Benefits}

This section attempts to quantify the ecosystem services that a GR can provide. Figure 5 indicates that HTC improvement and runoff reduction are two GR benefits being researched much more often than others during the last decade. Air quality improvement and noise reduction have been studied the least, with only three and two papers, respectively, out of the 102 papers reviewed in the present study. Additionally, further research on energy use reduction, runoff quality improvement, and ecological, social, and economic benefits are also required, as they were insufficiently studied with 22,18 , and 14 papers, respectively. These findings imply an identical trend with those previously reported in the reviews of Vijayaraghavan [2] and Shafique, Kim and Rafiq [1]. 


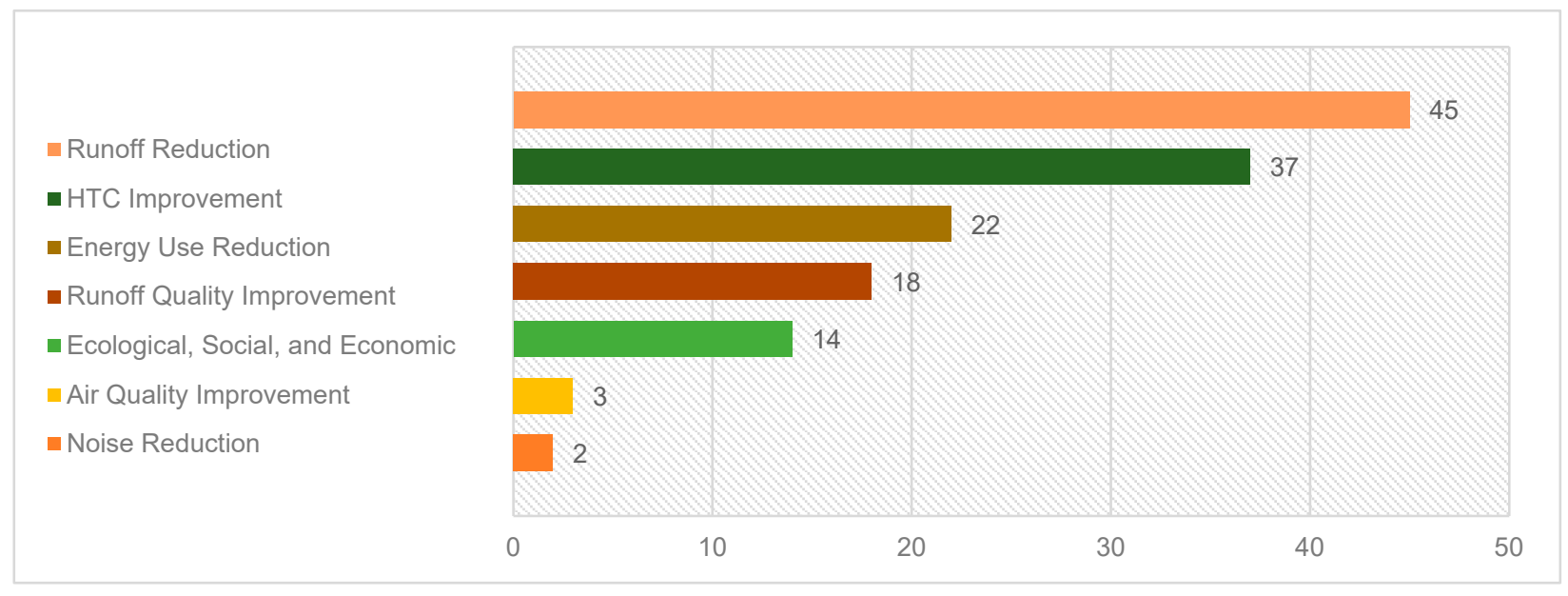

Figure 5. The break-down of papers as per the ecosystem services provided by GRs.

The above-discussed imbalance of GR research focus results from several factors. GR projects associated with temperature and runoff are easily carried out with the availability of numerous monitoring devices and existing simulation models. Consequently, such types of projects vary greatly, from indoor and outdoor experiments to model simulations. They can be also implemented in various methods comprising full-scale GRs, small test cells, and raised GR test beds. On the other hand, a lesser number of studies on runoff quality could be explained by the requirements of expensive instruments and advanced knowledge to collect and analyze water samples. Though there are 14 papers under the category "Ecological, Social, and Economic", only a small number of them have conducted the cost-benefit analysis to explore the costs of a GR system during its life cycle and its payback period. Meanwhile, fewer works have been done to study ecological and social benefits due to the difficulties in quantifying the recovery of habitat loss, the enhancement of urban biodiversity, and the improved human health and well-being by connecting people to green spaces $[1,3,38,39]$. By contrast, noise reduction and air pollution mitigation are two GR benefits that are receiving the least attention from researchers. This limitation could be due to the fact that these two benefits require a complex research design and specific knowledge relating to plant biochemistry. In particular, the amount of $\mathrm{CO}_{2}$, a primary greenhouse gas, that is directly absorbed by GR plants is minimal in comparison with indirect $\mathrm{CO}_{2}$ reduction from the energy savings provided by GRs [21].

This review referred to the Köppen climate classification system so as to understand the impact of climatic conditions on GR performance. This system divides the global climate into five main groups. The first letters, including A, B, C, D, and E, represent five types of climate: Tropical, Dry, Temperate, Continental, and Polar, respectively. To further describe these climate types, the second and third letters help to divide them into numerous sub-groups based on the precipitation and temperature characteristics. For instance, a subgroup Cfa (known as a humid sub-tropical climate) has the following features: Temperate (C), No dry season (f), and Hot summer (a). The following sub-sections give detailed information about how each of the GR benefits have been studied during last ten years.

\subsection{Runoff Reduction}

GR has been well known as an effective WSUD treatment due to its capability of holding precipitation in its layers, thus reducing stress on urban drainage systems. The hydrological behaviours of GR vary significantly because of several factors. Among those, rainfall depth appears to be the most influential one. The impact of rainfall depth on the GR retention rate could be identified not only by comparing results between different papers but also by considering individual papers. For example, cumulative rainfall retention by GRs reached a peak of $82.9 \%$ among 46 identified papers in Table 1 because a rainfall depth 
of only $628.2 \mathrm{~mm}$, ranging from 0.8 to $78.8 \mathrm{~mm}$ per event, was recorded during the study period of 15 months [40]. Another high retention rate of $73 \%$ was observed in the study of Zhang et al. [41] as only $563.7 \mathrm{~mm}$ of precipitation during 468 days was monitored. Others reported a smaller amount of rainfall retained greatly by GRs due to significant rainfall events and higher accumulated rainfall depths during the monitoring periods. Some examples are $45.1 \%$ [22], 50.2\% [42], and 51.4\% [43], corresponding to cumulative rainfall depths of $1256.3 \mathrm{~mm}$ (1 year), $1892.2 \mathrm{~mm}$ (27 months), and $481 \mathrm{~mm}$ (5 months), respectively. The lowest retention rate of $11.9 \%$ in the study of Wong and Jim [44] was due to the exposure of the GR to heavy rainfall events (more than $300 \mathrm{~mm}$ per event) with a total depth of $1102.7 \mathrm{~mm}$ in a short study period of 10 months.

Taking runoff reduction, rainfall retention from a single event, and peak flow reduction into account, these parameters demonstrate an identical pattern with the cumulative rainfall retention discussed in the above paragraph. Such a hydrological GR response has been mentioned in plenty of previous reports. Carson, Marasco, Culligan and McGillis [10] found a downward trend in the rainfall retention rate as precipitation increases. Palermo, Turco, Principato and Piro [22], Todorov et al. [45], Speak et al. [46], and Stovin, Vesuviano and Kasmin [42] found that the retention performance of GRs, when analyzing all events, was higher than the retention rate when considering only storm events. On an event-by-event basis, Zhang et al. [47] found an average retention rate of $95 \%$, as only small rainfall events, ranging from 2- to 35.2-mm depths, took place during the study period. Following the study of Todorov, Driscoll and Todorova [45] and Carpenter et al. [48], the retention rates of $95.9 \%$ and $96.8 \%$ corresponded with rainfall depths ranging between 0.76 and $44.2 \mathrm{~mm}$ and 2.5 to $17.8 \mathrm{~mm}$, respectively. Other papers applying large rainfall volumes showed lower retention capacities. Zhang et al. [49], Nawaz et al. [50], Hakimdavar et al. [51], Wong and Jim [44], and Stovin, Vesuviano and Kasmin [42] are good examples. Table 1 summarizes the hydrological performance of GRs from 45 studies. The results from this review are in agreement with those presented in Zheng, Zou, Lounsbury, Wang, Wang and Recycling [9], namely, that the rainfall volume retained during a single event ranges from 0 to $100 \%$ with an average value of $62 \%$. 
Table 1. Quantification of runoff reduction caused by green roofs.

\begin{tabular}{|c|c|c|c|c|c|c|c|c|c|}
\hline Number & Reference & Climate Group & Modelling Software Used & $\begin{array}{l}\text { Rainfall Depth } \\
\text { (mm) (Rainfall } \\
\text { Intensity (mm/h)) }\end{array}$ & $\begin{array}{c}\text { Runoff Reduction } \\
\qquad \%)\end{array}$ & $\begin{array}{l}\text { Cumulative } \\
\text { Rainfall Retention } \\
(\%)\end{array}$ & $\begin{array}{l}\text { Rainfall Retention } \\
\text { per Single Rainfall } \\
\text { Event (\%) }\end{array}$ & $\begin{array}{l}\text { Peak Flow } \\
\text { Reduction per } \\
\text { Single Rainfall } \\
\text { Event }(\%)\end{array}$ & $\begin{array}{c}\text { Rainfall } \\
\text { Retention from } \\
\text { Storm Events }\end{array}$ \\
\hline \multirow{2}{*}{1} & \multirow{2}{*}{ Barnhart et al. [52] } & \multirow{2}{*}{$\mathrm{Cfb}$} & \multirow{2}{*}{$\begin{array}{l}\text { Visualizing Ecosystem Land } \\
\text { Management Assessments } \\
\text { (VELMA) }\end{array}$} & \multirow{2}{*}{$\mathrm{N} / \mathrm{A}$} & EGR: 10-15 (annual) & \multirow{2}{*}{ N/A } & \multirow{2}{*}{$\mathrm{N} / \mathrm{A}$} & \multirow{2}{*}{$\mathrm{N} / \mathrm{A}$} & \multirow{2}{*}{ N/A } \\
\hline & & & & & IGR: 20-25 (annual) & & & & \\
\hline 2 & $\begin{array}{l}\text { Baek, Ligaray, } \\
\text { Pachepsky, Chun, } \\
\text { Yoon, Park and } \\
\text { Cho [27] }\end{array}$ & Cfa and Cwa & SWWM and HYDRUS-1D & $\mathrm{N} / \mathrm{A}$ & $\mathrm{N} / \mathrm{A}$ & $\mathrm{N} / \mathrm{A}$ & $\mathrm{N} / \mathrm{A}$ & $\mathrm{N} / \mathrm{A}$ & $\mathrm{N} / \mathrm{A}$ \\
\hline 4 & $\begin{array}{l}\text { Liu, Sun, Niu and } \\
\text { Riley [20] }\end{array}$ & Cwa & SWWM & 30 to 70 & 27 to 42 & N/A & 41 to 75 & 8 to 31 & $\mathrm{~N} / \mathrm{A}$ \\
\hline \multirow{2}{*}{5} & \multirow{2}{*}{ Jahanfar et al. [54] } & \multirow{2}{*}{ Dfa } & \multirow{2}{*}{$\mathrm{N} / \mathrm{A}$} & \multirow{2}{*}{ less than 10} & \multirow{2}{*}{$\mathrm{N} / \mathrm{A}$} & \multirow{2}{*}{$\mathrm{N} / \mathrm{A}$} & $\begin{array}{l}\text { Control GR: } 90 \\
\text { (minimum) }\end{array}$ & \multirow{2}{*}{ N/A } & \\
\hline & & & & & & & $\begin{array}{l}\text { PV GR: } 61 \text { to } 75 \\
\text { (minimum) }\end{array}$ & & \\
\hline 6 & $\begin{array}{l}\text { Zhang, Szota, Fletcher, } \\
\text { Williams and } \\
\text { Farrell [47] }\end{array}$ & $\mathrm{Cfb}$ & $\mathrm{N} / \mathrm{A}$ & 2 to 35.2 & $\mathrm{~N} / \mathrm{A}$ & $\mathrm{N} / \mathrm{A}$ & 89 to 95 (average) & $\mathrm{N} / \mathrm{A}$ & $\mathrm{N} / \mathrm{A}$ \\
\hline 8 & $\begin{array}{l}\text { Palermo, Turco, } \\
\text { Principato and } \\
\text { Piro [22] }\end{array}$ & Csa & HYDRUS-1D & $\begin{array}{l}2 \text { to } 120 \text {, and } 1256.3 \\
\text { (1 year) }\end{array}$ & $\mathrm{N} / \mathrm{A}$ & 45.1 (1 year) & $\begin{array}{l}16.7 \text { to } 100 \\
\text { (68 average) }\end{array}$ & $\begin{array}{l}13.3 \text { to } 95.2 \\
\text { (56 average) }\end{array}$ & $\begin{array}{c}16.7 \text { to } 82.5 \\
\text { (49.6 average) }\end{array}$ \\
\hline 9 & $\begin{array}{c}\text { Gong, Yin, Li, Zhang, } \\
\text { Wang, Fang, Shi and } \\
\text { Wang [40] }\end{array}$ & Dwa & $\mathrm{N} / \mathrm{A}$ & $\begin{array}{c}0.8 \text { to } 78.8 \text {, and } 628.2 \\
\text { (15 months) }\end{array}$ & $\mathrm{N} / \mathrm{A}$ & $\begin{array}{l}68.5 \text { to } 82.9 \\
\text { (15 months) }\end{array}$ & $\begin{array}{l}12.1 \text { to } 100 \text {, and ( } 88.1 \\
\text { to } 92.9 \text { average) }\end{array}$ & 72.3 to 95.9 & $\mathrm{~N} / \mathrm{A}$ \\
\hline 10 & Talebi et al. [56] & $\begin{array}{l}\text { Cfb, Dwb, Dfa, and } \\
\text { Dfb }\end{array}$ & $\begin{array}{l}\text { Penman-Monteith (PM) model } \\
\text { and Hargreaves and Samani } \\
\text { (HS) model }\end{array}$ & 390 to 1200 (annual) & $\begin{array}{l}17 \text { to } 47 \text { and } 27 \text { to } 61 \\
\text { (annual, low and } \\
\text { high water-use } \\
\text { plants, respectively) }\end{array}$ & $\mathrm{N} / \mathrm{A}$ & $\mathrm{N} / \mathrm{A}$ & $\mathrm{N} / \mathrm{A}$ & N/A \\
\hline
\end{tabular}


Table 1. Cont.

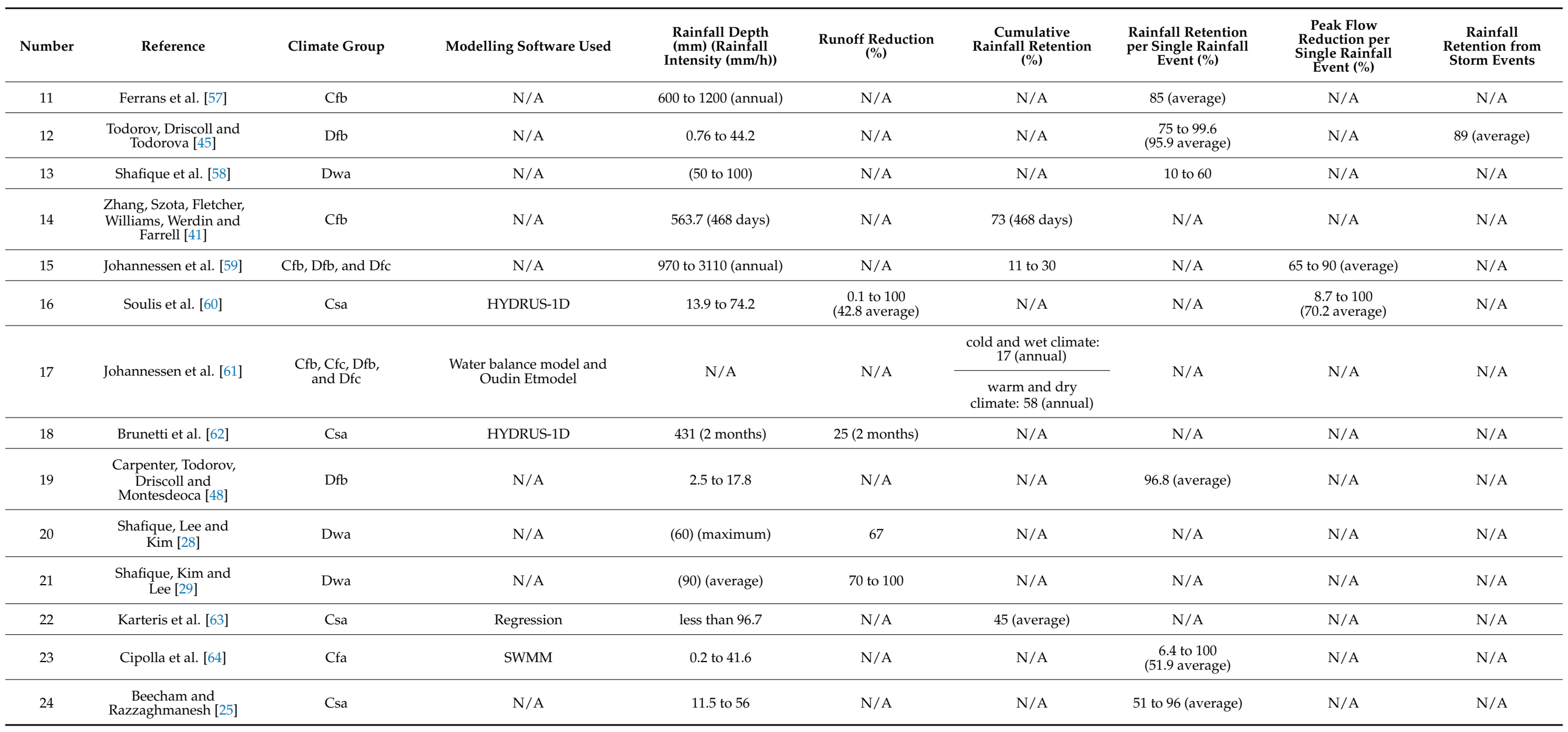


Table 1. Cont.

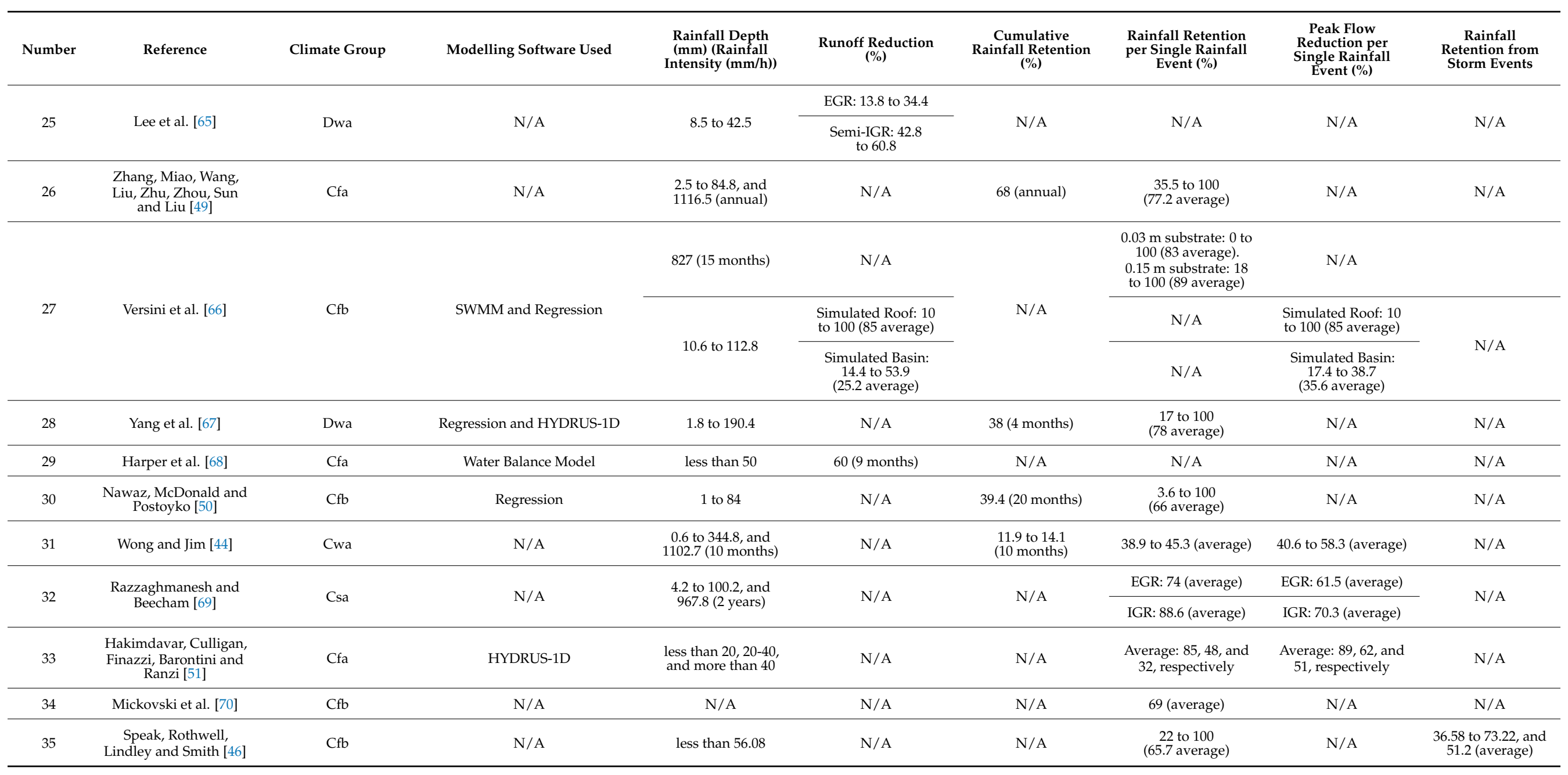


Table 1. Cont.

\begin{tabular}{|c|c|c|c|c|c|c|c|c|c|}
\hline Number & Reference & Climate Group & Modelling Software Used & $\begin{array}{l}\text { Rainfall Depth } \\
\text { (mm) (Rainfall } \\
\text { Intensity (mm/h)) }\end{array}$ & $\begin{array}{c}\text { Runoff Reduction } \\
(\%)\end{array}$ & $\begin{array}{c}\begin{array}{c}\text { Cumulative } \\
\text { Rainfall Retention } \\
(\%)\end{array} \\
\text { (1) }\end{array}$ & $\begin{array}{l}\text { Rainfall Retention } \\
\text { per Single Rainfall } \\
\text { Event (\%) }\end{array}$ & $\begin{array}{c}\text { Peak Flow } \\
\text { Reduction per } \\
\text { Single Rainfall } \\
\text { Event (\%) }\end{array}$ & $\begin{array}{c}\text { Rainfall } \\
\text { Retention from } \\
\text { Storm Events }\end{array}$ \\
\hline 36 & $\begin{array}{l}\text { Carson, Marasco, } \\
\text { Culligan and } \\
\text { McGillis [10] }\end{array}$ & $\mathrm{Cfa}$ & Regression & 0.25 to 180 & $\mathrm{~N} / \mathrm{A}$ & $\begin{array}{l}36,47, \text { and } 61 \\
\text { (1 year) }\end{array}$ & $\begin{array}{l}3 \text { to } 100,9 \text { to } 100 \\
\text { and } 20 \text { to } 100\end{array}$ & $\mathrm{~N} / \mathrm{A}$ & $\mathrm{N} / \mathrm{A}$ \\
\hline 37 & $\begin{array}{l}\text { Nagase and Dunnett } \\
\text { [71] }\end{array}$ & $\mathrm{Cfb}$ & $\mathrm{N} / \mathrm{A}$ & N/A & $\mathrm{N} / \mathrm{A}$ & $\mathrm{N} / \mathrm{A}$ & $\mathrm{N} / \mathrm{A}$ & $\mathrm{N} / \mathrm{A}$ & $\mathrm{N} / \mathrm{A}$ \\
\hline 38 & $\begin{array}{c}\text { Stovin, Vesuviano and } \\
\text { Kasmin [42] }\end{array}$ & $\mathrm{Cfb}$ & Regression & 1892.2 (27 months) & N/A & 50.2 (27 months) & 0 to 100 (70 average) & 60 (average) & $\begin{array}{c}0 \text { to } 100 \text {, and } 43 \\
\text { (average) }\end{array}$ \\
\hline 39 & Qin et al. [72] & Af & N/A & 18 & N/A & N/A & 11.4 & 65 & N/A \\
\hline \multirow[b]{2}{*}{40} & \multirow{2}{*}{$\begin{array}{l}\text { Buccola and } \\
\text { Spolek [73] }\end{array}$} & \multirow[b]{2}{*}{ Csb } & \multirow[b]{2}{*}{ N/A } & Heavy: (340) & \multirow[b]{2}{*}{ N/A } & \multirow[b]{2}{*}{ N/A } & Heavy: 56 (average) & \multirow[b]{2}{*}{ N/A } & \multirow[b]{2}{*}{ N/A } \\
\hline & & & & Medium: (30) & & & $\begin{array}{l}\text { Medium: } 64 \\
\text { (average) }\end{array}$ & & \\
\hline 41 & Beck et al. [74] & $\mathrm{Csb}$ & N/A & (74) & $\mathrm{N} / \mathrm{A}$ & N/A & $\begin{array}{c}8.5 \text { to } 32.5 \\
\text { (21.1 average) }\end{array}$ & N/A & N/A \\
\hline 42 & $\begin{array}{l}\text { Gregoire and } \\
\text { Clausen [43] }\end{array}$ & Dfa & Water Balance Model & 481 (5 months) & $\mathrm{N} / \mathrm{A}$ & 51.4 (5 months) & $\mathrm{N} / \mathrm{A}$ & N/A & N/A \\
\hline 44 & Voyde et al. [76] & $\mathrm{Cfb}$ & N/A & 1093 (1 year) & N/A & 66 (1 year) & 82 (average) & 93 (average) & N/A \\
\hline \multirow{2}{*}{45} & \multirow{2}{*}{ Palla et al. [77] } & \multirow{2}{*}{$\mathrm{Cfa}$} & \multirow{2}{*}{$\mathrm{N} / \mathrm{A}$} & \multirow{2}{*}{$\begin{array}{l}\text { Laboratory test: (108 } \\
\text { to 194). Field } \\
\text { experiment: } 8 \\
\text { to } 138.2\end{array}$} & \multirow{2}{*}{$\mathrm{N} / \mathrm{A}$} & \multirow{2}{*}{$\mathrm{N} / \mathrm{A}$} & $\begin{array}{l}\text { Laboratory test: } 52 \\
\text { to } 67 \\
\end{array}$ & \multirow{2}{*}{$\begin{array}{l}\text { Field experiment: } 44 \\
\text { to } 100 \text { (83.3 average) }\end{array}$} & \multirow{2}{*}{$\mathrm{N} / \mathrm{A}$} \\
\hline & & & & & & & $\begin{array}{l}\text { Field experiment: } 0 \\
\text { to } 100 \text { (51.5 average) }\end{array}$ & & \\
\hline
\end{tabular}


In order to make a proper comparison between different climatic locations, some papers simulated GRs with identical configurations to identify their hydrological behaviours in various weather patterns. Talebi, Bagg, Sleep and O'Carroll [56] recognized the best performance of GR in Regina (Dfb) and Calgary (Dwb), which received the least precipitation (390 and $420 \mathrm{~mm}$, respectively) among six studied Canadian cities, whereas the worst performance was observed in Halifax (Dfb), which had an average annual precipitation of $1400 \mathrm{~mm}$. In the study of Johannessen, Muthanna and Braskerud [59], a GR in Bergen $(\mathrm{Cfb})$ with $3110 \mathrm{~mm}$ of annual precipitation could retain total rainfall at only $11 \%$, whereas the accumulated retention rate in Trondheim (Dfc) was 30\%. Johannessen, Hanslin and Muthanna [61] considered ten northern European cities. They concluded that the wettest climate (Bergen, $\mathrm{Cfb}$ ) showed the lowest annual retention rate of $17 \%$; meanwhile, the driest climate (Malmö, $\mathrm{Cfb}$ ) achieved the highest rate of $58 \%$.

\subsection{Human Thermal Comfort (HTC) Improvement}

Although GRs are an important WSUD strategy, they also help to address temperatureand heat-related urban issues. The decrease in outer surface temperature $\left(\mathrm{T}_{\mathrm{S}}\right)$ of a roof deck after GR installation widely fluctuates, between around $5^{\circ} \mathrm{C}$ and $40{ }^{\circ} \mathrm{C}$, with a reduction of $15{ }^{\circ} \mathrm{C}$ to $20{ }^{\circ} \mathrm{C}$ reported the most (Table 2 The difference in $\mathrm{T}_{\mathrm{s}}$ between a GR and a bare roof $\left(\Delta \mathrm{T}_{\mathrm{S}}=\mathrm{T}_{\mathrm{s} \text {-bare roof }}-\mathrm{T}_{\mathrm{s}-\mathrm{GR}}\right)$ was the highest, at $40{ }^{\circ} \mathrm{C}$ in the study of He et al. [78], whereas Sun et al. [79] observed the smallest $\Delta \mathrm{T}_{\mathrm{s}}$ of $4.2^{\circ} \mathrm{C}$. The variation of a GRs' thermal response depends on many factors, such as the structural design of GRs and the thermal properties of GR materials, and the different climatic conditions that the GR is exposed to. However, some identical patterns, as well as noticeable discrepancies, have been identified in this review.

More specifically, in many studies, while GRs produced the most impressive results in hot climates, they showed the weakest impacts in cold climatic conditions. For instance, following $\mathrm{He}, \mathrm{Yu}$, Ozaki and Dong [18], a GR reduced $\mathrm{T}_{\mathrm{s}}$ by $21.7^{\circ} \mathrm{C}$ and $14.4^{\circ} \mathrm{C}$ in summer and winter, respectively. He et al. [80] also reported similar results with the $\mathrm{T}_{\mathrm{s}}$ reduction of $35^{\circ} \mathrm{C}$ and $15^{\circ} \mathrm{C}$ in the hottest and coldest seasons, respectively. Furthermore, in studies considering the impact of climatic conditions on thermal performance, GRs in hot and dry climates demonstrated the highest $\Delta \mathrm{T}_{\mathrm{s}}$. For example, Ávila-Hernández, Simá, Xamán, Hernández-Pérez, Téllez-Velázquez and Chagolla-Aranda [19] and Morakinyo, Dahanayake, Ng and Chow [26] utilized a computer-based tool to test the thermal effectiveness of GRs in different climates. They both found that cities in hot desert climates, which are indicated by the BWh sub-group, had the most positive results relative to those in other locations. It was also determined that the GRs in continental climates (Dfa or Dwa) achieved the poorest performance with regard to $\Delta T_{S}[30,79,81]$. Shanghai, China (Dfa) was found the most-appropriate location to implement GRs, as the greatest $\Delta T_{S}$ was recorded in this city $[78,80,82]$.

Table 2. Quantification of Human Thermal Comfort improvement by green roofs.

\begin{tabular}{|c|c|c|c|c|c|c|}
\hline Number & Reference & Climate Group & $\begin{array}{l}\text { Modelling } \\
\text { Software Used }\end{array}$ & $\begin{array}{c}\text { Surface } \\
\text { Temperature } \\
\text { Reduction- }-\Delta \mathrm{T}_{\mathrm{S}} \\
\left({ }^{\circ} \mathrm{C}\right)\end{array}$ & $\begin{array}{l}\text { Indoor Air } \\
\text { Temperature } \\
\text { Reduction- } \\
\Delta \mathrm{T}_{\text {air.in }} \\
\quad\left({ }^{\circ} \mathrm{C}\right)\end{array}$ & $\begin{array}{c}\text { Outdoor Air } \\
\text { Temperature } \\
\text { Reduction- } \\
\Delta \mathrm{T}_{\text {air.out }} \\
\quad\left({ }^{\circ} \mathrm{C}\right)\end{array}$ \\
\hline 1 & $\begin{array}{l}\text { La Roche, Yeom and } \\
\text { Ponce [36] }\end{array}$ & Csa & Regression & $\mathrm{N} / \mathrm{A}$ & $\begin{array}{l}2.1 \text { (averaged } \\
\text { maximum, } \\
\text { compared with } \\
\text { insulated bare } \\
\text { roof) }\end{array}$ & $\mathrm{N} / \mathrm{A}$ \\
\hline 2 & $\begin{array}{c}\text { Ávila-Hernández, Simá, } \\
\text { Xamán, } \\
\text { Hernández-Pérez, } \\
\text { Téllez-Velázquez and } \\
\text { Chagolla-Aranda [19] }\end{array}$ & $\begin{array}{l}\text { As }(\mathrm{Aw}), \mathrm{Am}, \\
\text { BSh, BWh, BSk, } \\
\text { and Cwb }\end{array}$ & EnergyPlus & 14.5 (maximum) & $\begin{array}{c}\text { Maximum: } 4.7 \\
\text { (upper level) and } \\
0.9 \text { (lower level) }\end{array}$ & $\mathrm{N} / \mathrm{A}$ \\
\hline
\end{tabular}


Table 2. Cont.

\begin{tabular}{|c|c|c|c|c|c|c|}
\hline Number & Reference & Climate Group & $\begin{array}{l}\text { Modelling } \\
\text { Software Used }\end{array}$ & $\begin{array}{c}\text { Surface } \\
\text { Temperature } \\
\text { Reduction- }-\Delta \mathrm{T}_{\mathrm{S}} \\
\left({ }^{\circ} \mathrm{C}\right)\end{array}$ & $\begin{array}{c}\text { Indoor Air } \\
\text { Temperature } \\
\text { Reduction- } \\
\Delta \mathrm{T}_{\text {air.in }} \\
\left({ }^{\circ} \mathrm{C}\right)\end{array}$ & $\begin{array}{c}\text { Outdoor Air } \\
\text { Temperature } \\
\text { Reduction- } \\
\Delta \mathrm{T}_{\text {air.out }} \\
\quad\left({ }^{\circ \mathrm{C})}\right.\end{array}$ \\
\hline 3 & $\begin{array}{l}\text { Feitosa and Wilkinson } \\
\text { [35] }\end{array}$ & Cfa & $\mathrm{N} / \mathrm{A}$ & $\mathrm{N} / \mathrm{A}$ & $\begin{array}{l}\text { WGBT: }-1.9 \\
\text { (nighttime) and } \\
8.3 \text { (daytime) }\end{array}$ & $\mathrm{N} / \mathrm{A}$ \\
\hline \multirow{2}{*}{4} & \multirow{2}{*}{$\begin{array}{l}\text { He, Yu, Ozaki and } \\
\text { Dong [18] }\end{array}$} & \multirow{2}{*}{ Cfa } & \multirow{2}{*}{ THERB } & $\begin{array}{c}\text { Summer } \\
\text { maximum: } 21.7 \\
\text { (daytime) and } \\
-5.3 \text { (nighttime) }\end{array}$ & \multirow{2}{*}{$\mathrm{N} / \mathrm{A}$} & \multirow{2}{*}{$\mathrm{N} / \mathrm{A}$} \\
\hline & & & & $\begin{array}{l}\text { Winter maximum: } \\
14.4 \text { (daytime) } \\
\text { and }-9.2 \\
\text { (nighttime) }\end{array}$ & & \\
\hline 5 & $\begin{array}{c}\text { Xing, Hao, Lin, Tan and } \\
\text { Yang [33] }\end{array}$ & Cfa & $\mathrm{N} / \mathrm{A}$ & $\mathrm{N} / \mathrm{A}$ & $\begin{array}{l}-3 \text { (maximum, } \\
\text { nighttime) }\end{array}$ & $\mathrm{N} / \mathrm{A}$ \\
\hline 6 & Cao et al. [83] & Cfa & $\mathrm{N} / \mathrm{A}$ & $\begin{array}{l}\text { Maximum: } 11.9 \\
\text { (compared to bare } \\
\text { soil roof) }\end{array}$ & $\mathrm{N} / \mathrm{A}$ & $\mathrm{N} / \mathrm{A}$ \\
\hline 7 & Tang and Zheng [84] & Cfa & $\mathrm{N} / \mathrm{A}$ & 16.4 (maximum) & 3.1 (average) & $\mathrm{N} / \mathrm{A}$ \\
\hline \multirow{2}{*}{8} & \multirow{2}{*}{ Cai et al. [85] } & \multirow{2}{*}{ Cfa } & \multirow{2}{*}{$\mathrm{N} / \mathrm{A}$} & $\begin{array}{c}\text { Summer: } 5.7 \\
\text { (average, sunny) }\end{array}$ & $\begin{array}{l}\text { Summer: } 0.6 \\
\text { (average, sunny) }\end{array}$ & \multirow{2}{*}{$\mathrm{N} / \mathrm{A}$} \\
\hline & & & & $\begin{array}{l}\text { Winter: }-1.2 \\
\text { (average, sunny) }\end{array}$ & $\begin{array}{l}\text { Winter: }-1.6 \\
\text { (average, sunny) }\end{array}$ & \\
\hline 9 & $\begin{array}{c}\text { Cascone, Catania, } \\
\text { Gagliano and } \\
\text { Sciuto [21] }\end{array}$ & Csa & EnergyPlus & 19 (maximum) & $\mathrm{N} / \mathrm{A}$ & $\mathrm{N} / \mathrm{A}$ \\
\hline \multirow{2}{*}{10} & \multirow{2}{*}{$\begin{array}{c}\text { Feitosa and } \\
\text { Wilkinson [34] }\end{array}$} & \multirow{2}{*}{ Aw and Cfa } & \multirow{2}{*}{$\mathrm{N} / \mathrm{A}$} & \multirow{2}{*}{$\mathrm{N} / \mathrm{A}$} & $\begin{array}{l}\text { Rio de Janeiro, } \\
\text { maximum WGBT: } \\
8.1 \text { (daytime) and } \\
-2.8 \text { (nighttime) }\end{array}$ & \multirow{2}{*}{$\mathrm{N} / \mathrm{A}$} \\
\hline & & & & & $\begin{array}{c}\text { Sydney, } \\
\text { maximum WGBT: } \\
12 \text { (daytime) and } \\
-1.2 \text { (nighttime) }\end{array}$ & \\
\hline 11 & Park et al. [86] & Dwa & Regression & $\mathrm{N} / \mathrm{A}$ & $\mathrm{N} / \mathrm{A}$ & $\begin{array}{c}\text { Maximum, } 1.5 \mathrm{~m} \\
\text { above: } 22.6 \\
\text { (daytime) and } 1.9 \\
\text { ( nighttime) }\end{array}$ \\
\hline 12 & Lee and Jim [23] & Cwa & $\mathrm{N} / \mathrm{A}$ & 19.8 (maximum) & $\mathrm{N} / \mathrm{A}$ & $\begin{array}{c}\text { Maximum: } 6.21 \\
(0.15 \mathrm{~m} \text { above }), \\
4.7(0.50 \mathrm{~m} \text { above, } \\
\text { and } 3.1(1.5 \mathrm{~m} \\
\text { above })\end{array}$ \\
\hline 13 & Azeñas et al. [87] & Csa & $\mathrm{N} / \mathrm{A}$ & $\mathrm{N} / \mathrm{A}$ & $\mathrm{N} / \mathrm{A}$ & $\mathrm{N} / \mathrm{A}$ \\
\hline \multirow{2}{*}{14} & \multirow{2}{*}{$\begin{array}{c}\text { Morakinyo, } \\
\text { Dahanayake, Ng and } \\
\text { Chow [26] }\end{array}$} & \multirow{2}{*}{$\begin{array}{l}\text { BWh, Cfa, Cwa, } \\
\text { and } \mathrm{Cfb}\end{array}$} & \multirow{2}{*}{$\begin{array}{l}\text { EnergyPlus and } \\
\text { ENVI-met }\end{array}$} & \multirow{2}{*}{$\begin{array}{l}\text { Maximum: } 14 \\
\text { (daytime) and } 4 \\
\quad \text { (nighttime) }\end{array}$} & $\begin{array}{c}\text { Maximum, hot } \\
\text { climate, } 0.7-\mathrm{m} \\
\text { substrate: } 1.4 \\
\text { (daytime) and } 0.3 \\
\text { (nighttime) }\end{array}$ & \multirow{2}{*}{$\begin{array}{c}\text { Maximum, } 1.5 \mathrm{~m} \\
\text { above, pedestrian } \\
\text { level: } 0.6 \\
\text { (daytime) and } \\
-0.2 \text { (nighttime) }\end{array}$} \\
\hline & & & & & $\begin{array}{l}\text { Maximum, cold } \\
\text { climate, } 0.3-\mathrm{m} \\
\text { substrate: } 0.3 \\
\text { (daytime) and } \\
-0.1 \text { (nighttime) }\end{array}$ & \\
\hline
\end{tabular}


Table 2. Cont.

\begin{tabular}{|c|c|c|c|c|c|c|}
\hline Number & Reference & Climate Group & $\begin{array}{l}\text { Modelling } \\
\text { Software Used }\end{array}$ & $\begin{array}{c}\text { Surface } \\
\text { Temperature } \\
\text { Reduction }-\Delta \mathrm{T}_{\mathrm{S}} \\
\left({ }^{\circ} \mathrm{C}\right)\end{array}$ & $\begin{array}{c}\text { Indoor Air } \\
\text { Temperature } \\
\text { Reduction- } \\
\Delta \mathrm{T}_{\text {air.in }} \\
\left({ }^{\circ} \mathrm{C}\right)\end{array}$ & $\begin{array}{c}\text { Outdoor Air } \\
\text { Temperature } \\
\text { Reduction- } \\
\Delta \mathrm{T}_{\text {air.out }} \\
\left({ }^{\circ} \mathrm{C}\right)\end{array}$ \\
\hline \multirow{2}{*}{15} & \multirow{2}{*}{$\begin{array}{l}\text { He, Yu, Ozaki, Dong } \\
\text { and Zheng [80] }\end{array}$} & \multirow{2}{*}{ Cfa } & \multirow{2}{*}{$\begin{array}{l}\text { A coupled heat } \\
\text { and moisture } \\
\text { transfer model }\end{array}$} & $\begin{array}{c}\text { Summer } \\
\text { maximum: } 35 \\
\text { (daytime) and }-5 \\
\text { (nighttime) }\end{array}$ & \multirow{2}{*}{$\mathrm{N} / \mathrm{A}$} & \multirow{2}{*}{$\begin{array}{c}\text { Maximum, } 0.15 \mathrm{~m} \\
\text { above: } 5 \\
\text { (summer) and } 2 \\
\text { (winter) }\end{array}$} \\
\hline & & & & $\begin{array}{l}\text { Winter maximum: } \\
15 \text { (daytime) and } \\
-10 \text { (nighttime) }\end{array}$ & & \\
\hline 16 & Yeom and La Roche [37] & Csa & $\mathrm{N} / \mathrm{A}$ & $\mathrm{N} / \mathrm{A}$ & $\begin{array}{l}2 \text { (compared to } \\
\text { other test cells) }\end{array}$ & $\mathrm{N} / \mathrm{A}$ \\
\hline 17 & Shafique and Kim [30] & Dwa & $\mathrm{N} / \mathrm{A}$ & 5 to 9 (average) & $\mathrm{N} / \mathrm{A}$ & $\mathrm{N} / \mathrm{A}$ \\
\hline \multirow{2}{*}{18} & \multirow{2}{*}{ Wilkinson et al. [88] } & \multirow{2}{*}{ Aw and $\mathrm{Cfa}$} & \multirow{2}{*}{$\mathrm{N} / \mathrm{A}$} & \multirow{2}{*}{$\mathrm{N} / \mathrm{A}$} & $\begin{array}{l}\text { Rio de Janeiro } \\
\text { maximum: }-4.1 \\
\text { (nighttime) and } \\
6.2 \text { (daytime) }\end{array}$ & \multirow{2}{*}{$\mathrm{N} / \mathrm{A}$} \\
\hline & & & & & $\begin{array}{c}\text { Sydney } \\
\text { maximum: -1.1 } \\
\text { (nighttime) and } \\
12 \text { (daytime) }\end{array}$ & \\
\hline 19 & $\begin{array}{l}\text { Bevilacqua, Mazzeo, } \\
\text { Bruno and Arcuri [82] }\end{array}$ & Csa & $\mathrm{N} / \mathrm{A}$ & $\begin{array}{l}\text { Maximum: } 34.1 \\
\text { (daytime) and } \\
-9.4 \text { (nighttime) }\end{array}$ & $\mathrm{N} / \mathrm{A}$ & $\mathrm{N} / \mathrm{A}$ \\
\hline 20 & Foustalieraki et al. [89] & Csa & $\mathrm{N} / \mathrm{A}$ & $\begin{array}{l}\text { Maximum: } 21.9 \\
\text { (daytime) and } \\
-1.6 \text { (nighttime) }\end{array}$ & $\begin{array}{l}\text { Maximum: } 1.1 \\
\text { (summer) and } \\
-0.7 \text { (winter) }\end{array}$ & $\mathrm{N} / \mathrm{A}$ \\
\hline 21 & $\begin{array}{l}\text { Boafo, Kim and } \\
\text { Kim [81] }\end{array}$ & Dwa & EnergyPlus & $\begin{array}{l}\text { Maximum: } 5 \\
\text { (summer) and }-6 \\
\text { (winter) }\end{array}$ & $\mathrm{N} / \mathrm{A}$ & $\mathrm{N} / \mathrm{A}$ \\
\hline 22 & Gagliano et al. [90] & Csa & $\begin{array}{l}\text { The Design } \\
\text { Builder software }\end{array}$ & 19 (maximum) & 4 (maximum) & $\mathrm{N} / \mathrm{A}$ \\
\hline 23 & $\begin{array}{c}\text { Shafique, Kim and } \\
\text { Lee [29] }\end{array}$ & Dwa & $\mathrm{N} / \mathrm{A}$ & 10 (maximum) & $\mathrm{N} / \mathrm{A}$ & $\mathrm{N} / \mathrm{A}$ \\
\hline 24 & $\begin{array}{c}\mathrm{He}, \mathrm{Yu}, \text { Dong and } \\
\text { Ye [78] }\end{array}$ & Cfa & $\mathrm{N} / \mathrm{A}$ & $\begin{array}{c}\text { Maximum: } 30 \\
\text { (free floating) and } \\
40 \text { (air } \\
\text { conditioned) }\end{array}$ & $\begin{array}{l}\text { Maximum: } 2 \\
\text { (daytime) and } \\
-2.5 \text { (nighttime) }\end{array}$ & $\begin{array}{l}5 \text { (maximum, } \\
0.15 \mathrm{~m} \text { above) }\end{array}$ \\
\hline 25 & Tam et al. [91] & Cwa & $\mathrm{N} / \mathrm{A}$ & $\mathrm{N} / \mathrm{A}$ & 3.4 (maximum) & $\mathrm{N} / \mathrm{A}$ \\
\hline 26 & $\begin{array}{l}\text { Schweitzer and } \\
\text { Erell [92] }\end{array}$ & Csa & $\mathrm{N} / \mathrm{A}$ & $\mathrm{N} / \mathrm{A}$ & $\begin{array}{c}1.89 \text { (average) } \\
\text { and } 4.5 \\
\text { (maximum) }\end{array}$ & $\mathrm{N} / \mathrm{A}$ \\
\hline 27 & $\begin{array}{l}\text { Chemisana and } \\
\text { Lamnatou [93] }\end{array}$ & BSk & $\mathrm{N} / \mathrm{A}$ & 14 (maximum) & $\mathrm{N} / \mathrm{A}$ & $\mathrm{N} / \mathrm{A}$ \\
\hline 28 & $\begin{array}{l}\text { Sun, Bou-Zeid, Wang, } \\
\text { Zerba and Ni [79] }\end{array}$ & Dwa and Dfa & PROM & $\begin{array}{l}4.2 \text { (summer, aver- } \\
\text { aged daily } \\
\text { maximum) }\end{array}$ & $\mathrm{N} / \mathrm{A}$ & $\mathrm{N} / \mathrm{A}$ \\
\hline 29 & Peng and Jim [3] & Cwa & $\begin{array}{l}\text { ENVI-met and } \\
\text { RayMan }\end{array}$ & $\mathrm{N} / \mathrm{A}$ & $\begin{array}{c}\text { Maximum: } 1.6 \\
\text { (Top Floor) and } \\
1.3 \text { (Ground } \\
\text { Floor) }\end{array}$ & $\begin{array}{c}\text { Maximum, } 1.2 \mathrm{~m} \\
\text { above: } 2.1 \\
\text { (rooftop level) } \\
\text { and } 1.7 \\
\text { (pedestrian level) }\end{array}$ \\
\hline 30 & $\begin{array}{c}\text { Ascione, Bianco, } \\
\text { de'Rossi, Turni and } \\
\text { Vanoli [16] }\end{array}$ & $\begin{array}{c}\text { Bsh, Csa, Cfb, and } \\
\text { Dfb }\end{array}$ & EnergyPlus & $\mathrm{N} / \mathrm{A}$ & $\mathrm{N} / \mathrm{A}$ & $\mathrm{N} / \mathrm{A}$ \\
\hline
\end{tabular}


Table 2. Cont.

\begin{tabular}{|c|c|c|c|c|c|c|}
\hline Number & Reference & Climate Group & $\begin{array}{l}\text { Modelling } \\
\text { Software Used }\end{array}$ & $\begin{array}{c}\text { Surface } \\
\text { Temperature } \\
\text { Reduction }-\Delta \mathrm{T}_{\mathrm{S}} \\
\left({ }^{\circ} \mathrm{C}\right)\end{array}$ & $\begin{array}{c}\text { Indoor Air } \\
\text { Temperature } \\
\text { Reduction- } \\
\Delta \mathrm{T}_{\text {air.in }} \\
\left({ }^{\circ} \mathrm{C}\right)\end{array}$ & $\begin{array}{c}\text { Outdoor Air } \\
\text { Temperature } \\
\text { Reduction- } \\
\Delta \mathrm{T}_{\text {air.out }} \\
\quad\left({ }^{\circ} \mathrm{C}\right)\end{array}$ \\
\hline 31 & Pandey et al. [94] & Cwa & $\mathrm{N} / \mathrm{A}$ & $\mathrm{N} / \mathrm{A}$ & $\begin{array}{l}\text { Average: } 3.9 \\
\text { (DBT) and } 4 \\
\text { (WGBT) }\end{array}$ & $\mathrm{N} / \mathrm{A}$ \\
\hline 32 & Blanusa et al. [95] & $\mathrm{Cfb}$ & $\mathrm{N} / \mathrm{A}$ & $\begin{array}{l}\text { Compared with } \\
\text { bare soil roof: } \\
14.9 \text { (maximum) }\end{array}$ & $\mathrm{N} / \mathrm{A}$ & $\begin{array}{l}\text { Compared with } \\
\text { bare soil roof: } 1.1 \\
\text { (average, } 0.1 \mathrm{~m} \\
\text { above) }\end{array}$ \\
\hline 33 & $\begin{array}{c}\text { Qin, Wu, Chiew and } \\
\text { Li [72] }\end{array}$ & Af & $\mathrm{N} / \mathrm{A}$ & $\begin{array}{l}15.3 \text { (maximum) } \\
\text { and } 7.3 \text { (average) }\end{array}$ & $\mathrm{N} / \mathrm{A}$ & $\begin{array}{c}0.3 \mathrm{~m} \text { above: } 1.3 \\
\text { (maximum) and } \\
0.5 \text { (average) }\end{array}$ \\
\hline 34 & Jim and Peng [96] & Cwa & $\mathrm{N} / \mathrm{A}$ & 12.5 (maximum) & $\mathrm{N} / \mathrm{A}$ & $\begin{array}{c}\text { Maximum: } 4.4 \\
(0.1 \mathrm{~m} \text { above }) \text { and } \\
2.3 \text { (1.6 } \mathrm{m} \text { above })\end{array}$ \\
\hline 35 & Pérez et al. [97] & Bsk & $\mathrm{N} / \mathrm{A}$ & $\mathrm{N} / \mathrm{A}$ & $\mathrm{N} / \mathrm{A}$ & $\mathrm{N} / \mathrm{A}$ \\
\hline 36 & Getter et al. [98] & Dfa & $\mathrm{N} / \mathrm{A}$ & 20 (maximum) & $\mathrm{N} / \mathrm{A}$ & $\mathrm{N} / \mathrm{A}$ \\
\hline 37 & Hui and Chan [99] & Cwa & $\mathrm{N} / \mathrm{A}$ & $\begin{array}{c}4 \text { to } 5 \text { (compared } \\
\text { to non-PV GR) }\end{array}$ & $\mathrm{N} / \mathrm{A}$ & $\mathrm{N} / \mathrm{A}$ \\
\hline
\end{tabular}

Owing to the interaction between the surface temperature of the roof membrane and the indoor environment, $\left(\mathrm{T}_{\text {air.in }}\right), \Delta \mathrm{T}_{\text {air.in }}\left(\mathrm{T}_{\text {air.in-bare roof }}-\mathrm{T}_{\text {air.in-GR }}\right)$, and $\Delta \mathrm{T}_{\mathrm{S}}$ demonstrate a similar behaviour. However, $\Delta \mathrm{T}_{\text {air.in }}$ is much smaller than $\Delta \mathrm{T}_{\mathrm{S}}$, with respect to both magnitude and amplitude. In accordance with the data presented in Table $2, \Delta \mathrm{T}_{\text {air.in }}$ ranged from around $1{ }^{\circ} \mathrm{C}$ to $5^{\circ} \mathrm{C}$. The integration of GRs and green walls significantly improved


respectively [88]. In alignment with $\mathrm{T}_{\mathrm{S}}$, the $\mathrm{T}_{\text {air.in }}$ of a living roof was mostly reported as warmer than that of a bare roof during nighttime and wintertime. GRs acting as a heat source could be ideal to reduce heating demands in cold regions, but this is not the case in hot climatic regions.

The ability of GRs to mitigate urban heat island (UHI) effects has been inadequately examined. Of the 37 published works in Table 2, there are few papers researching the decrease in ambient air temperature $\left(\Delta \mathrm{T}_{\text {air.out }}\right)$ after GR implementation. Some exceptions are the studies by Park, Kim, Dvorak and Lee [86], Lee and Jim [23], Morakinyo, Dahanayake, $\mathrm{Ng}$ and Chow [26], Peng and Jim [3], and Jim and Peng [96], as they undertook an analysis of $\mathrm{T}_{\text {air.out }}$ above a living roof and a non-living roof. Nevertheless, in addition to different GR characteristics and study locations, they produced greatly divergent outcomes due to the nonidentical set-ups of their measuring devices. He, Yu, Ozaki, Dong and Zheng [80] concluded that the GR was able to reduce air temperature at $0.15 \mathrm{~m}$ above the roof by $5{ }^{\circ} \mathrm{C}$ in summer and $2{ }^{\circ} \mathrm{C}$ in winter. Lee and Jim [23] set thermal sensors to monitor $\mathrm{T}_{\text {air.out }}$ above the studied roofs at three positions, including $0.15 \mathrm{~m}, 0.5 \mathrm{~m}$, and $1.5 \mathrm{~m}$. Qin, Wu, Chiew and $\mathrm{Li}$ [72] and Peng and Jim [3] reported values of $\mathrm{T}_{\text {air.out }}$ at $0.3 \mathrm{~m}$ and $1.2 \mathrm{~m}$ above the roofs, respectively. Park, Kim, Dvorak and Lee [86] reported an outstanding result, as $\Delta \mathrm{T}_{\text {air.out }}$ reached a peak of $22.6^{\circ} \mathrm{C}$, whereas others observed much smaller $\mathrm{T}_{\text {air.out }}$ reductions. However, it was similarly detected that a higher reduction was observed closer to plant canopies and the greater effect of GRs occurred during the day and in summer. Moreover, only two papers estimated $\Delta \mathrm{T}_{\text {air.out }}$ at the pedestrian level after growing plants on building rooftops [3,26]. Future studies similar to that by Köhler and Kaiser [17] are important, since they conducted a comprehensive investigation of GR performance with regard to UHI mitigation with a 20 -year monitoring period. They found that although the ambient temperature had an upward trend due to global warming, the temperature of the GR substrate layer remained stable with a slight decrease. Consequently, the poten- 
tial of GRs to address the UHI effects is still optimistic, and sufficient studies need to be undertaken with an identical approach prior to making generic conclusions.

\subsection{Energy Use Reduction}

In accordance with thermal reduction, GR reduces the energy that a building consumes for cooling and heating demand. Twenty-two papers were identified in the present study that reported varying results in terms of savings in energy use. However, some similarities can also be observed in Table 3. All publications pointed out a higher decrease in cooling demand than heating demand after implementation of GRs. For example, Cai, Feng, $\mathrm{Yu}$, Xiang and Chen [85] achieved an annual reduction of $11.2 \%$ and $9.3 \%$ of energy consumption for cooling and heating, respectively. The difference was even larger in Cascone, Catania, Gagliano and Sciuto [21]; the yearly amount of electricity consumed by a GR-integrated building was 35\% (cooling) and 10\% (heating) less than a building with a conventional roof. Moreover, these two values in Gagliano, Detommaso, Nocera and Berardi [90] were impressive, with maximum decreases of $85 \%$ and $48 \%$ for cooling and heating requirements, respectively.

Table 3. Quantification of energy use reduction caused by green roofs.

\begin{tabular}{|c|c|c|c|c|c|c|}
\hline \multirow{2}{*}{ Number } & \multirow{2}{*}{ Reference } & \multirow{2}{*}{ Climate Group } & \multirow{2}{*}{$\begin{array}{c}\text { Modelling } \\
\text { Software Used }\end{array}$} & \multicolumn{3}{|c|}{ Energy Performance } \\
\hline & & & & $\begin{array}{c}\text { Energy } \\
\text { Reduction }\end{array}$ & $\begin{array}{l}\text { Heat Flux (HF) } \\
\text { Reduction }-\Delta H F\end{array}$ & $\begin{array}{l}\mathrm{CO}_{2} \text { Emission } \\
\text { Reduction }\end{array}$ \\
\hline 1 & $\begin{array}{c}\text { Ávila-Hernández, Simá, } \\
\text { Xamán, } \\
\text { Hernández-Pérez, } \\
\text { Téllez-Velázquez and } \\
\text { Chagolla-Aranda [19] }\end{array}$ & $\begin{array}{l}\text { As }(\mathrm{Aw}), \mathrm{Am}, \\
\text { BSh, BWh, BSk, } \\
\text { and Cwb }\end{array}$ & EnergyPlus & $\begin{array}{l}\text { Maximum: } 99 \% \\
\text { (cooling) and } \\
-25 \% \text { (heating) }\end{array}$ & $\mathrm{N} / \mathrm{A}$ & $\begin{array}{l}2.5 \text { tons or } 45.7 \% \\
\text { (maximum, } \\
\text { annual) }\end{array}$ \\
\hline \multirow[t]{2}{*}{2} & \multirow[t]{2}{*}{$\begin{array}{c}\mathrm{He}, \mathrm{Yu}, \text { Ozaki and } \\
\text { Dong [18] }\end{array}$} & \multirow[t]{2}{*}{ Cfa } & \multirow[t]{2}{*}{ THERB } & \multirow{2}{*}{$\begin{array}{l}\text { Top floor: } 3.6 \% \\
\text { (cooling) and } \\
6.2 \% \text { (heating) }\end{array}$} & $\begin{array}{c}\text { Summer } \\
\text { maximum: } 12.6 \\
\text { (daytime) and } \\
-3.1 \text { (nighttime) } \\
\mathrm{W} / \mathrm{m}^{2}\end{array}$ & \multirow[t]{2}{*}{$\mathrm{N} / \mathrm{A}$} \\
\hline & & & & & $\begin{array}{c}\text { Winter maximum: } \\
8.4 \text { (daytime) and } \\
-5.4 \text { (nighttime) } \\
\mathrm{W} / \mathrm{m}^{2}\end{array}$ & \\
\hline 3 & $\begin{array}{c}\text { Xing, Hao, Lin, Tan and } \\
\text { Yang [33] }\end{array}$ & Cfa & $\mathrm{N} / \mathrm{A}$ & $18 \%$ (heating) & $\begin{array}{l}3.1 \mathrm{~W} / \mathrm{m}^{2} \\
\text { (average, heating } \\
\text { condition) }\end{array}$ & $\mathrm{N} / \mathrm{A}$ \\
\hline 4 & Tang and Zheng [84] & $\mathrm{Cfa}$ & $\mathrm{N} / \mathrm{A}$ & $\begin{array}{l}\text { 14.7\% (average, } \\
\text { cooling) }\end{array}$ & $\begin{array}{c}35.5 \mathrm{~W} / \mathrm{m}^{2} \\
\text { (maximum, } \\
\text { daytime) and } \\
76.1 \% \text { (average) }\end{array}$ & $\mathrm{N} / \mathrm{A}$ \\
\hline \multirow{2}{*}{5} & \multirow{2}{*}{$\begin{array}{l}\text { Cai, Feng, Yu, Xiang } \\
\text { and Chen [85] }\end{array}$} & \multirow{2}{*}{$\mathrm{Cfa}$} & \multirow{2}{*}{ Swell BESI2016 } & \multirow{2}{*}{$\begin{array}{c}\text { Annual: } 10.13 \% \\
\text { (total), } 9.3 \% \\
\text { (heating), and } \\
11.2 \% \text { (cooling) }\end{array}$} & $\begin{array}{l}\text { Summer: } 3.7 \\
\text { W/ } \mathrm{m}^{2} \text { or } 50 \% \\
\text { (average, } \\
\text { daytime) }\end{array}$ & \multirow{2}{*}{$\begin{array}{c}9.35 \mathrm{~kg} / \mathrm{m}^{2} \mathrm{GR} \\
\text { (annual) }\end{array}$} \\
\hline & & & & & $\begin{array}{c}\text { Winter: } \\
-7.5 \mathrm{~W} / \mathrm{m}^{2} \text { or } \\
24.6 \% \text { (average, } \\
\text { daytime) }\end{array}$ & \\
\hline
\end{tabular}


Table 3. Cont.

\begin{tabular}{|c|c|c|c|c|c|c|}
\hline \multirow[b]{2}{*}{ Number } & \multirow[b]{2}{*}{ Reference } & \multirow[b]{2}{*}{ Climate Group } & \multirow{2}{*}{$\begin{array}{c}\text { Modelling } \\
\text { Software Used }\end{array}$} & \multicolumn{3}{|c|}{ Energy Performance } \\
\hline & & & & $\begin{array}{l}\text { Energy } \\
\text { Reduction }\end{array}$ & $\begin{array}{l}\text { Heat Flux (HF) } \\
\text { Reduction }-\Delta \mathrm{HF}\end{array}$ & $\begin{array}{l}\mathrm{CO}_{2} \text { Emission } \\
\text { Reduction }\end{array}$ \\
\hline 6 & $\begin{array}{c}\text { Cascone, Catania, } \\
\text { Gagliano and } \\
\text { Sciuto [21] }\end{array}$ & Csa & FASST & $\begin{array}{l}\text { Annual: } 20-24 \% \\
\text { (total), 31-35\% } \\
\text { (cooling), and } \\
2-10 \% \text { (heating) }\end{array}$ & $\mathrm{N} / \mathrm{A}$ & $\begin{array}{c}1.35 \mathrm{~kg} / \mathrm{m}^{2} \mathrm{GR} \\
\text { (annual) }\end{array}$ \\
\hline 7 & $\begin{array}{c}\text { Azeñas, Cuxart, Picos, } \\
\text { Medrano, Simó, } \\
\text { López-Grifol and } \\
\text { Gulías [87] }\end{array}$ & Csa & $\mathrm{N} / \mathrm{A}$ & $\mathrm{N} / \mathrm{A}$ & $\begin{array}{l}48 \text { to } 86 \% \\
\text { (annual) }\end{array}$ & $\mathrm{N} / \mathrm{A}$ \\
\hline 8 & $\begin{array}{c}\text { Morakinyo, } \\
\text { Dahanayake, Ng and } \\
\text { Chow [26] }\end{array}$ & $\begin{array}{l}\text { BWh, Cfa, Cwa, } \\
\text { and } \mathrm{Cfb}\end{array}$ & EnergyPlus & $\begin{array}{l}\text { Maximum. } \\
\text { cooling: } 5.2 \% \text { (hot } \\
\text { climate, } 0.7-\mathrm{m} \text { soil } \\
\text { thickness) and } \\
0.3 \% \text { (temperate } \\
\text { climate, } 0.3-\mathrm{m} \text { soil } \\
\text { thickness) }\end{array}$ & $\mathrm{N} / \mathrm{A}$ & $\mathrm{N} / \mathrm{A}$ \\
\hline 9 & $\begin{array}{l}\text { He, Yu, Ozaki, Dong } \\
\text { and Zheng [80] }\end{array}$ & Cfa & $\begin{array}{l}\text { A coupled heat } \\
\text { and moisture } \\
\text { transfer model }\end{array}$ & $\mathrm{N} / \mathrm{A}$ & $\begin{array}{c}\text { Average: } 1.75 \\
\mathrm{~W} / \mathrm{m}^{2} \text { (summer) } \\
\text { and } 0.87 \mathrm{~W} / \mathrm{m}^{2} \\
\text { (winter) }\end{array}$ & $\mathrm{N} / \mathrm{A}$ \\
\hline 10 & $\begin{array}{l}\text { Foustalieraki, } \\
\text { Assimakopoulos, } \\
\text { Santamouris and } \\
\text { Pangalou [89] }\end{array}$ & Csa & EnergyPlus & $\begin{array}{l}\text { Annual: } 15.1 \% \\
\text { (total), } 18.7 \% \\
\text { (cooling), and } \\
11.4 \% \text { (heating) }\end{array}$ & $\mathrm{N} / \mathrm{A}$ & $\mathrm{N} / \mathrm{A}$ \\
\hline 11 & $\begin{array}{l}\text { Boafo, Kim and } \\
\text { Kim [81] }\end{array}$ & Dwa & EnergyPlus & $\begin{array}{l}\text { Annual: } 3.7 \% \\
\text { (total), } 5.4 \% \\
\text { (cooling), and } \\
2.7 \% \text { (heating) }\end{array}$ & $\mathrm{N} / \mathrm{A}$ & $\mathrm{N} / \mathrm{A}$ \\
\hline 12 & $\begin{array}{c}\text { Karteris, Theodoridou, } \\
\text { Mallinis, Tsiros and } \\
\text { Karteris [63] }\end{array}$ & Csa & EnergyPlus & $\begin{array}{c}\text { Maximum: } 5 \% \\
\text { (heating) and } 16 \% \\
\text { (cooling) }\end{array}$ & $\mathrm{N} / \mathrm{A}$ & $\begin{array}{c}3.5 \text { to } 9.1 \mathrm{~kg} / \mathrm{m}^{2} \\
\text { GR (annual) }\end{array}$ \\
\hline 13 & $\begin{array}{l}\text { Gagliano, Detommaso, } \\
\text { Nocera and Berardi [90] }\end{array}$ & Csa & $\begin{array}{l}\text { The Design } \\
\text { Builder software }\end{array}$ & $\begin{array}{l}\text { Maximum: } 85 \% \\
\text { (cooling) and } 48 \% \\
\text { (heating) }\end{array}$ & $\mathrm{N} / \mathrm{A}$ & $\mathrm{N} / \mathrm{A}$ \\
\hline 14 & $\begin{array}{c}\mathrm{He}, \mathrm{Yu}, \text { Dong and } \\
\text { Ye [78] }\end{array}$ & Cfa & $\mathrm{N} / \mathrm{A}$ & $\mathrm{N} / \mathrm{A}$ & $\begin{array}{c}\text { Maximum } \\
\text { daytime: } 15 \text { (free } \\
\text { floating) and } 20 \\
\text { (air conditioning) } \\
\mathrm{W} / \mathrm{m}^{2}\end{array}$ & $\mathrm{~N} / \mathrm{A}$ \\
\hline 15 & $\begin{array}{l}\text { Schweitzer and Erell } \\
\text { [92] }\end{array}$ & Csa & $\mathrm{N} / \mathrm{A}$ & $\mathrm{N} / \mathrm{A}$ & $\begin{array}{c}679 \mathrm{~kJ} / \mathrm{m}^{2} \\
\text { (average) }\end{array}$ & $\mathrm{N} / \mathrm{A}$ \\
\hline 16 & $\begin{array}{l}\text { Sun, Bou-Zeid, Wang, } \\
\text { Zerba and Ni [79] }\end{array}$ & Dwa and Dfa & PROM & $\mathrm{N} / \mathrm{A}$ & $\begin{array}{c}133 \mathrm{~W} / \mathrm{m}^{2} \\
\text { (averaged daily } \\
\text { maximum) }\end{array}$ & $\mathrm{N} / \mathrm{A}$ \\
\hline 17 & $\begin{array}{c}\text { Ascione, Bianco, } \\
\text { de'Rossi, Turni and } \\
\text { Vanoli [16] }\end{array}$ & $\begin{array}{c}\text { Bsh, Csa, Cfb, and } \\
\text { Dfb }\end{array}$ & EnergyPlus & $\begin{array}{l}\text { Maximum: } 11 \% \\
\text { (warm climate) } \\
\text { and } 7 \% \text { (cold } \\
\text { climate) }\end{array}$ & $\mathrm{N} / \mathrm{A}$ & $\mathrm{N} / \mathrm{A}$ \\
\hline 18 & $\begin{array}{l}\text { Pandey, Hindoliya and } \\
\text { Mod [94] }\end{array}$ & Cwa & $\mathrm{N} / \mathrm{A}$ & $\mathrm{N} / \mathrm{A}$ & $\begin{array}{c}13.8 \mathrm{~W} / \mathrm{m}^{2} \text { and } \\
73.8 \% \text { (maximum, } \\
\text { daytime) }\end{array}$ & $\mathrm{N} / \mathrm{A}$ \\
\hline 19 & Jim and Peng [96] & Cwa & $\mathrm{N} / \mathrm{A}$ & $\begin{array}{c}2.8 \times 10^{4} \mathrm{kWh} \\
\left(\text { cooling, } 484 \mathrm{~m}^{2}\right. \\
\text { GR) }\end{array}$ & $\begin{array}{c}33.5 \mathrm{~W} / \mathrm{m}^{2} \\
\text { (maximum, } \\
\text { daytime) }\end{array}$ & $\begin{array}{l}27.02 \text { tons } \\
\text { (summer, at } \\
\text { power plant) }\end{array}$ \\
\hline 20 & $\begin{array}{c}\text { Pérez, Coma, Solé, } \\
\text { Castell and Cabeza [97] }\end{array}$ & Bsk & $\mathrm{N} / \mathrm{A}$ & $\begin{array}{c}3.6 \text { to } 15 \% \\
\text { (cooling) and } \\
-7 \% \text { (heating) }\end{array}$ & $\mathrm{N} / \mathrm{A}$ & $\mathrm{N} / \mathrm{A}$ \\
\hline
\end{tabular}


Table 3. Cont.

\begin{tabular}{|c|c|c|c|c|c|c|}
\hline \multirow{2}{*}{ Number } & \multirow{2}{*}{ Reference } & \multirow{2}{*}{ Climate Group } & \multirow{2}{*}{$\begin{array}{l}\text { Modelling } \\
\text { Software Used }\end{array}$} & \multicolumn{3}{|c|}{ Energy Performance } \\
\hline & & & & $\begin{array}{c}\text { Energy } \\
\text { Reduction }\end{array}$ & $\begin{array}{l}\text { Heat Flux (HF) } \\
\text { Reduction- }-\Delta \mathrm{HF}\end{array}$ & $\begin{array}{c}\mathrm{CO}_{2} \text { Emission } \\
\text { Reduction }\end{array}$ \\
\hline 21 & $\begin{array}{l}\text { Getter, Rowe, Andresen } \\
\text { and Wichman [98] }\end{array}$ & Dfa & $\mathrm{N} / \mathrm{A}$ & $\mathrm{N} / \mathrm{A}$ & $\begin{array}{l}\text { Average: } 167 \% \\
\text { (summer) and } \\
13 \% \text { (winter) }\end{array}$ & $\mathrm{N} / \mathrm{A}$ \\
\hline 22 & Hui and Chan [99] & Cwa & EnergyPlus & $\begin{array}{c}6.53 \times 10^{4} \mathrm{kWh} \\
\left(6300 \mathrm{~m}^{2} \mathrm{GR}\right. \\
\text { annual })\end{array}$ & $\mathrm{N} / \mathrm{A}$ & N/A \\
\hline
\end{tabular}

In a broader context, some papers modelled buildings with vegetated roofs in various climates to investigate the climatic influence on the energy performance of GRs. Overall, the energy effectiveness of GRs has reported the greatest savings in hot climates and the least savings in cold climates. Ascione, Bianco, de'Rossi, Turni and Vanoli [16] reported a maximum reduction of $11 \%$ in warm climates, whereas $7 \%$ was estimated in cold climates. Morakinyo, Dahanayake, Ng and Chow [26] fulfilled a comprehensive analysis of energy reduction due to GRs with different substrate depths and climatic conditions. As expected, GRs with a deeper substrate $(0.7 \mathrm{~m})$ in hot climates (BWh) outperformed GRs with a thinner substrate $(0.3 \mathrm{~m})$ in temperate climates $(\mathrm{Cfb})$, with respect to energy savings for cooling at $5.2 \%$ and $0.3 \%$, respectively. Ávila-Hernández, Simá, Xamán, Hernández-Pérez, TéllezVelázquez and Chagolla-Aranda [19] brought a different perspective by investigating the energy behavior of a residential building with a living roof in several Mexican cities. The largest annual energy savings, of more than $90 \%$, was recorded in cities where the cooling demand exceeds the heating demand. In spite of the large energy reduction for cooling, those cities consumed more energy for heating because the cooling effect of the GR was inappropriate for low-average-annual-temperature regions (BSk and Cwb). Finally, the simulation results suggested that GRs should be implemented in warm-climate cities (Am and $\mathrm{BWh}$ ), which are dominated by cooling demand.

Another parameter used in the studies is heat flux (HF), which is defined as the flow of energy through building envelopes. Many papers considered HF because of its strong correlation with the electricity consumption of a building. In this context, a GR acts as an insulation layer to reduce heat flux moving into a building through the rooftop. This reduction of energy flow $\left(\Delta \mathrm{HF}=\mathrm{HF}_{\text {bare roof }}-\mathrm{HF}_{\mathrm{GR}}\right)$ is in agreement with the $\Delta \mathrm{T}_{\text {air.in }}$ already discussed earlier. More accurately, $\Delta \mathrm{HF}$ was greater during daytime and hot summers than that during night-time and cold winters. There was a decrease of more than $50 \%$ in HF passing through the roof deck under a GR as compared to a conventional roof. The $\triangle \mathrm{HF}$ in Getter, Rowe, Andresen and Wichman [98] reached an average of $167 \%$ in summer, but only $13 \%$ in winter. In the study of He, Yu, Ozaki, Dong and Zheng [80], GRs lessened $\mathrm{HF}$ by $1.75 \mathrm{~W} / \mathrm{m}^{2}$ and $0.87 \mathrm{~W} / \mathrm{m}^{2}$ in summer and winter, respectively.

Few studies investigating building energy reduction due to GRs also studied the reduction of cardon dioxide $\left(\mathrm{CO}_{2}\right)$ concentration caused by a reduction in energy use. In addition to the direct $\mathrm{CO}_{2}$ absorption by GR plants, the indirect removal of $\mathrm{CO}_{2}$ is caused by the energy savings. The calculation of $\mathrm{CO}_{2}$ indirectly absorbed by GR vegetation was mostly based upon the emission factor and the amount of energy saved by the GR installation. Karteris, Theodoridou, Mallinis, Tsiros and Karteris [63] concluded that the implementation of a GR at the municipality level of Thessaloniki city could reduce 65,000 tons of $\mathrm{CO}_{2}$ yearly. Furthermore, the $\mathrm{CO}_{2}$ reduction generated by $50 \%$ of the building blocks in the city corresponds to the 50 -acre forest plantation. Simulation results from ÁvilaHernández, Simá, Xamán, Hernández-Pérez, Téllez-Velázquez and Chagolla-Aranda [19] show that applying GRs could save up to $45.7 \%$ of $\mathrm{CO}_{2}$ annually in Chetumal, which has a high cooling demand. In Wuxi, China, with a humid, subtropical climate (Cfa), each square meter of a GR could reduce $9.35 \mathrm{~kg}$ of $\mathrm{CO}_{2}$ per year [85]. 


\subsection{Runoff Quality Improvement}

In addition to runoff reduction, improving runoff quality is another ecosystem service provided by this WSUD strategy. However, this benefit requires further research due to the significant variation among the published results. Globally, researchers are trying to determine whether GRs enhance or degrade the stormwater quality. Several authors have been in agreement, with a conclusion that the runoff quality from GRs is strongly affected by the substrate composition (organic content), substrate depth, GR age, maintenance frequency, and fertilizing methods [1,2]. No standards exist for regulating the runoff quality from GRs [100]. Therefore, the vast majority of papers on runoff quality in this review used fresh-water standards from the American Public Health Association (APHA) and the United States Environmental Protection Agency (USEPA). A few papers used local guidelines to examine GR outflows, such as Razzaghmanesh et al. [101], with Australian drinking water guidelines and the South Australian Environment Protection Authority's policy on water quality.

Findings from 17 identified studies demonstrate the difference in GR performance associated with runoff quality. GRs performed inconsistently with different types of pollutants and GR systems. Gong, Yin, Li, Zhang, Wang, Fang, Shi and Wang [40] found that GRs reduced the loads of pollutants such as total phosphorus (TP) and total nitrogen (TN), but increased the load of chemical oxygen demand (COD). In contrast, different GR modules in Ferrans, Rey, Pérez, Rodríguez and Díaz-Granados [57] were effective for neutralizing the $\mathrm{pH}$ of rainwater, but were unsuccessful in absorbing most of the runoff pollutants, including TP, TN, and several metals. The majority of GR systems identified in this review were reportedly capable of increasing $\mathrm{pH}$ values of roof runoff to around 8 , a neutral value. Rainwater tends to be acidic, with average $\mathrm{pH}$ values of less than 5.6, and hence the neutralization of rainwater by GRs is an environmental benefit that prevents acidic runoff from flowing to receiving water bodies [102,103]. Harper, Limmer, Showalter and Burken [68] detected significant nutrient loads in GR discharge with a decreasing trend of concentration during the monitoring period. Zhang, Miao, Wang, Liu, Zhu, Zhou, Sun and Liu [49] found that GRs washed out ammonium nitrogen, total suspended solids (TSS), and fluoride anions, but was a source of the remaining pollutants. Additionally, the GR in the study by Vijayaraghavan and Raja [100] acted as a sink for all examined heavy metal ions during both un-spiked and spiked artificial rainfall events, whereas the GR was a source of all pollutants in the study by Beecham and Razzaghmanesh [25].

Among abovementioned variables, the composition of GR growing media and the use of fertilizers were found linked the most to the quality of GR runoff. For example, Beecham and Razzaghmanesh [25] pointed out that the outflows from GRs with higher organic matter had higher pollutant concentrations. The lower concentrations of TP and TN in the study of Gregoire and Clausen [43], as compared to other studies, were explained by the slow-release fertilizer, expanded shale, and biosolids media with a high sorption rate of pollutants. Zhang, Miao, Wang, Liu, Zhu, Zhou, Sun and Liu [49] stated that a high concentration of TN in GR discharge could result from the substrate used, which had a nitrogen-rich content. Harper, Limmer, Showalter and Burken [68] observed a greatly noticeable nutrient load in GR runoff due to the excessive nutrients contained in the substrate construction materials. All studied EGR modules of Gong, Yin, Li, Zhang, Wang, Fang, Shi and Wang [40] acted as a sink of TP and TN, but a source of COD. The significant release of COD had a strong connection to the substrate layer, as it contained plenty of turfy soil which had high contents of organic matter. Moreover, the pollutant loads of TP and TN in GR outflows were negligible because of no addition of nitrogen and phosphorus fertilizers.

Aside from the substrate materials and fertilizing practices, researchers also studied other factors potentially correlating to GR runoff quality. For example, Razzaghmanesh, Beecham and Kazemi [101] found that EGRs outperformed IGRs in terms of runoff quality because the thinner substrate of the EGRs restricted its capacity to leach pollutants from the soil media. The concentration of pollutants was the highest at the beginning of the GR 
operation and consecutively decreased until the end of study period, which is consistent with the finding of Harper, Limmer, Showalter and Burken [68]. Beecham and Razzaghmanesh [25] compared various types of roofs, including non-vegetated and vegetated beds of EGRs and IGRs. They concluded that non-vegetated EGRs and vegetated IGRs were more effective than non-vegetated IGRs and vegetated EGRs, respectively. While the former was due to the shallower substrate, the latter was from the plant uptake. The authors also highlighted the important role of vegetation in the pollutant removal efficiency. In addition, temperature was similarly reported in Buffam et al. [104] and Carpenter, Todorov, Driscoll and Montesdeoca [48] to be linked with the concentration of nutrients in the GR discharge. The quality of stormwater passing through GRs varied seasonally, with the highest concentration during the growing season and the greatest retention during the non-growing season. The mechanism behind this seasonal variation was that the higher temperature in the growing season was promoting the mineralization process in the soil media of the GRs.

\subsection{Ecological, Social, and Economic Benefits}

One of the biggest challenges confronting the application of GRs is the high installation costs and other costs arising from its operation. Although it is commonly understood that a GR system requires a considerable investment, it provides promising monetary savings after a short payback period as compared to conventional roofs [105]. The financial factor plays an important role in the successful acceptance of GRs since it facilitates approvals from policy makers, investors, and property owners. Therefore, the interest of researchers has also been directed towards economic benefits from the implementation of GRs. For instance, Ávila-Hernández, Simá, Xamán, Hernández-Pérez, Téllez-Velázquez and Chagolla-Aranda [19] made an economic evaluation by considering the electricity and irrigation costs. Their GR in the city of Hermosillo, Sonora could provide a return on investment after only 8.8 years. Cai, Feng, Yu, Xiang and Chen [85] studied the economic benefit of GRs by converting the GR ecosystem services into monetary values. More specifically, they took into account the carbon sequestration, oxygen release, air pollution mitigation, rainwater management, and energy savings. The computed net present value (NPV) showed that a GR could become profitable from the 10th year after its installation. With nearly identical variables, Cascone, Catania, Gagliano and Sciuto [21] performed an economic assessment based on an actual circumstance with a tax deduction of $65 \%$, provided by Italian laws, for GR installation costs. The results show that the payback period varied according to drainage materials and vegetation types. They were 13.4 years for sedum with perlite and 17.9 years for salvia on rubber crumb. On the contrary, some works reported opposite results. In the study of Ascione, Bianco, de'Rossi, Turni and Vanoli [16], cool roofs were preferable with respect to economic benefits. By analyzing the watering and energy costs, GRs required more than 100 years to cover the investment cost. GR costs were even impossible to be paid in some locations. Another study of Berto et al. [106] supported the economic effectiveness of cool roofs over GRs from the private investors' point of view. The contradictory conclusions from the cost assessments are attributed to two facts. The first is the limitation of efforts made to analyze the GR costs, so the knowledge about this GR benefit is not comprehensively understood [2]. The second constraint is the difficulty in quantifying some of the GR ecosystem services, which makes the life-cycle assessment of GR difficult to be justified [1]. Consequently, further studies involving as many GR advantages as possible in the cost-benefit analysis are required prior to making final conclusions. Regardless of all discussed uncertainties, GRs are still believed to be much more superior to other roof types. Berto, Stival and Rosato [106] suggested that without counting the unquantifiable benefits, it was sensibly deduced that GRs could bring huge profits as compared to cool roofs. Vijayaraghavan [2] maintained that the likelihood of profits from a GR system could overcome all of its weakness and losses.

GR is able to promote the urban ecology by attracting wildlife species and restoring the habitat loss caused by urban development [2,3]. Additionally, GR offers numerous 
social benefits that are advantageous for human health and liveability. The reduction of heat stress, noise pollution, and air pollution play a crucial role in improving human health and well-being. Furthermore, the green spaces generated by GRs improve urban aesthetics and establish recreational activities in urban areas, thus improving social connectivity [1]. Although the ecological and social benefits of GRs are foreseeable, research on them has been insufficient. This is in conformity with a small number of relevant papers identified in this review. The difficulty in measuring such benefits is an explanation for such limited research. The following attempts are noteworthy in this direction. Kratschmer, Kriechbaum and Pachinger [24] studied the correlation of the diversity and abundance of wild bees with GRs. They found that wild bee species were positively affected by floral resources and finer and deeper substrates. Rumble et al. [107] investigated the presence of microarthropods, bacteria, and fungi in GR materials. They observed their survival and the independent colonization during eleven months after the construction of the GR. The species existing in GRs were found linked to both GR construction components and natural communities. Mycorrhizal fungi, which is beneficial for the uptake of nutrients by the plants, was suggested to be commercially added to GR construction. MacIvor and Lundholm [38] also detected the existence of a broad range of insects from five studied IGRs. This finding could encourage the idea of using GRs to improve urban biodiversity. Whittinghill et al. [108] researched the potential of the social benefits of GRs by calculating the food production by EGR plants. Tomatoes, beans, cucumbers, basil, and chives were chosen, and they survived and produced an adequate yield. This horticultural method, which is well known as a part of urban farming, is a promising strategy to address critical social issues such as food security and job opportunities.

\subsection{Air Quality Improvement}

GRs bring benefits to the air quality in two distinct ways. The first way is the indirect reduction of $\mathrm{CO}_{2}$ emission from energy savings, which has been already discussed in an earlier section. The second way is through the stomata of GR vegetation to remove this greenhouse gas from the atmosphere [109]. In addition to $\mathrm{CO}_{2}$, GRs are capable of capturing several air pollutants, consisting of $\mathrm{O}_{3}, \mathrm{NO}_{2}, \mathrm{PM}_{10}$, and $\mathrm{SO}_{2}$. However, $\mathrm{CO}_{2}$ has attracted the most attention of researchers. This review found only three papers studying this benefit provided by GRs. This small number is not likely to represent all works completed; however, it could partially reflect the limitation of efforts and interest in this research area. For instance, the large-scale application of GRs in Thessaloniki led to a $\mathrm{CO}_{2}$ sequestration rate of 3951.52 tons per year, and a total annual $\mathrm{CO}_{2}$ saving of 12,441 tons [63]. The ability of sequestrating $\mathrm{CO}_{2}$ depends on the selection of GR plants, and it was determined that spices and aromatic plants outperformed grasses. However, this reduction was not considerable as compared to a year-round $\mathrm{CO}_{2}$ saving of 65,000 tons by reducing the air-conditioning demand. GRs contribute to diminishing the $\mathrm{CO}_{2}$ emission by consuming the air pollutants from the atmosphere; nevertheless, this is minimal, relative to the indirect reduction from energy savings [21]. Another study performed by Baraldi et al. [110] examined the GR capability of capturing the air pollutants. They conducted a holistic analysis concerning the physiological features of fifteen GR species comprising shrubs and herbaceous plants. The results reveal that the capacity of mitigating the air pollution varies according to the specific species. Furthermore, all tested species were also found favorable for urban sustainable development as they are not likely to form ozone. Li et al. [111] adopted a mixed methodology to research the effect of GRs on the fluctuation of $\mathrm{CO}_{2}$ concentration in the ambient atmosphere. According to the field measurement, chamber experiment, and computer simulation, the authors stated that a GR had a large potential to reduce $\mathrm{CO}_{2}$ emission by up to $2 \%$ on a sunny day. They also concluded that the $\mathrm{CO}_{2}$ absorbing rate greatly depends on the condition of GR plants, sunlight intensity, wind velocity, and GR position. 


\subsection{Noise Reduction}

Another ecosystem service of GR is to establish an acoustic barrier confronting the sound transmission from the outdoor environment to a building. Noise from vehicles and other anthropogenic activities influencing human health and well-being is regarded as noise pollution. GR functions as an additional sound insulation layer as well as an absorber of sound waves passing through the building envelopes. In this review, only two papers were found to investigate this GR benefit. Connelly and Hodgson [112] conducted both a field experiment and a purpose-built field laboratory to investigate the loss of sound transmission through vegetated and non-vegetated roofs. As expected, the difference in sound transmission between the conventional roof and the GR was up to 10, 20, and more than 20 decibels $(\mathrm{dB})$ in the low-, mid-, and high-frequency ranges, respectively. It was also noted that the thicker substrates and the deeper-root plants enhanced the noise reduction, whereas the substrate moisture content caused no major changes. Following Yang et al. [113], they computed the noise reduction at street level by installing a GR on a low-profiled structure. The results show that a GR could successfully attenuate the sound annoyance level by up to $9.5 \mathrm{db}$. Moreover, the substrate type and depth were found to be not as important as the whole GR system in terms of traffic noise mitigation. Similar to the air quality improvement, this review identified a very small number of papers associated with the noise reduction. Therefore, it could be reasonably deduced that this research area needs more attention.

\section{Discussion}

\subsection{GR Types}

As can be seen in Figure 3, the number of papers applying EGRs are 6-7 times more than those applying IGRs and SIGRs. It is well known that EGRs have plenty of advantages that have led to their widespread implementation. On the contrary, the implementation of IGRs faces many challenges, as they require high structural load bearing capacities and high amounts of maintenance. However, with the consideration of the capability of providing ecosystem services alone, IGRs remarkably outperform EGRs. It is also worth noting that SIGRs appear to have a combination of advantages taken from both EGRs and IGRs. Their growing media are thinner and lighter than that of IGRs, and they support a broader range of appropriate plants than what EGRs can. Therefore, SIGRs not only eliminate the disadvantages of IGRs and EGRs but also optimize the GR benefits. Further research should study the potential of intensive and semi-intensive types of GRs, so that they are not disregarded. More efforts to explore alternative materials for light-weight GR systems are also encouraged.

Attempts to establish hybrid GR-based experiments were found to be negligible in this review. Such hybrid GRs are highly recommended because of the encouraging results achieved by them. For example, Hui and Chan [99] found that the $T_{s}$ of a hybrid photovoltaic GR (PV GR) was $5{ }^{\circ} \mathrm{C}$ cooler than that of a traditional GR, due to the shading effect of the PV panels. An increase of $4.3 \%$ in power output from the PV panels also resulted from this combined system. Another example of the enhanced performance of hybrid GRs is provided by Chemisana and Lamnatou [93]. They found a maximum $\Delta \mathrm{T}_{\mathrm{s}}$ of $14{ }^{\circ} \mathrm{C}$ as well as a maximum increase of $3.33 \%$ in solar energy from the PV system. Yeom and La Roche [37] evaluated the combination of a GR and a radiant cooling system by setting up various test cells. They found a $2-{ }^{\circ} \mathrm{C}$ lower temperature inside the cell with a GR and radiant pipes as compared to GRs with and without an insulation layer. Additionally, the combination of GRs and green walls provide outstanding thermal and energy reductions as compared to stand-alone GR systems [33-35,88]. In an attempt to improve the runoff retention rate, a system integrating GRs with blue roofs was developed and the runoff outflow rates from the green-blue roof and a control roof were $0.1 \mathrm{~L} / \mathrm{s}$ and $0.3 \mathrm{~L} / \mathrm{s}$, respectively, in the study by Shafique, Lee and Kim [28]. It is worth noting that the outflows from the green-blue roof only occurred a few hours after the beginning of a rainfall event, which helps to alleviate the stress on the urban drainage system. 


\subsection{GR Benefits}

As mentioned in previous sections, urban heat mitigation and runoff reductions have been found to be the most-studied GR benefits. On the other hand, the mitigation of air pollution through the direct $\mathrm{CO}_{2}$ sequestration of GR plants is a GR benefit that has been inadequately investigated. A barrier to undertake research on this GR benefit is the limited options for the vegetation layer. While EGR keeps receiving the most attention during the last ten years due to several advantages, shallow-root plants in EGRs have a smaller capacity to capture gaseous pollutants and dust particles in the atmosphere [1,2]. Large plants, such as trees with deep roots, could absorb greater amounts of air pollutants, but face many challenges in GR construction and operation during its lifespan. Other than that, the insignificant quantity of $\mathrm{CO}_{2}$ sequestrated by GR plants could be an explanation for their lesser preference among researchers. Last but not least, the simplest way to conduct such projects is by utilizing the factor values already computed in the literature, which could not produce dependable results. Otherwise, the investigation of this benefit (reduction in air pollution) requires a complex experimental set-up and specific knowledge about the phytology of plants.

Noise reduction is also inadequately researched due to various constraints. One of them could be attributed to the fact that a GR system can only lessen the noise transmitted from traffic into the indoor environment if it is installed on a low-rise building [2]. Along with the rapid urbanization and population growth, multi-storey buildings are being constructed. Consequently, the GRs of both existing and new buildings are positioned far away from the ground level. This reduces the possibility of the application of GRs for noise reduction. Another difficulty could be the lack of standards for measuring the sound transmission through roofs [112]. Moreover, methods for testing the transmission loss are mainly developed for other building components, such as interior walls and exterior facades.

Finally, other GR benefits relating to runoff quality, the ecosystem, and social and economic benefits have been researched more often than the mitigation of noise and air pollution. However, they are still in experimental stages due to various constraints. For example, the question of whether GRs improve or degrade the runoff quality should be properly answered before proceeding to implementation. Additionally, the cost-benefit analysis of GRs pointed out remarkable long-term savings, and many experts have confirmed that the GR benefits outweigh their potential costs. Nevertheless, numerous important benefits were not considered in the economic evaluation because most of them are difficult to convert into monetary values. Consequently, this hinders researchers from carrying out such projects. Hence, future studies are suggested to be conducted with a well-designed approach.

\subsection{Innovative GR Construction Techniques and Materials}

In spite of unsolved issues, the potential of GRs remains remarkably huge. Considering only the GRs' capability of providing ecosystem services, most studies illustrate positive outcomes. In order to facilitate the widespread implementation of GR, it is suggested that future studies should be directed towards identifying optimal GR designs to satisfy not only the ecosystem services provided by GRs but also their affordability for installing, operating, and maintaining. According to the literature review, it was determined that there exist attempts to explore alternative GR materials. Some of them succeeded to enhance the GR benefits. For example, Carpenter, Todorov, Driscoll and Montesdeoca [48] obtained a noticeable averaged retention rate of $96.8 \%$, which exceeded the previously-reported values mainly due to the application of an effective drainage layer. Another high retention rate from Todorov, Driscoll and Todorova [45] was also a result of a drainage design that was never applied in other studies. Altering the substrate composition by adding biochar improved both the retention capacity and quality of the GR discharge [74]. On the other hand, the application of modelling software to simulate GR performance was observed during the last decade. Though the accuracy of simulation results compared well 
with actual field data, their application for the large-scale implementation of GRs is still negligible. The costs and benefits of large-scale implementation of GRs need to be clear to authorities and stakeholders, such as building owners, builders, and developers. Therefore, this is a recommendation for future research studies.

\subsection{Inconsistent Impact of Parameters on GR Performance}

It is not challenging to find studies on the benefits of GRs relating to UHI mitigation and energy reduction. These two benefits of GRs are quite apparent during the period of the strongest solar radiation (SR). Conversely, they are less desirable at night and in cold weather conditions. Moreover, seasonal and daily thermal behaviours of GRs are even more complicated. He, Yu, Ozaki and Dong [18], He, Yu, Ozaki, Dong and Zheng [80], Bevilacqua, Mazzeo, Bruno and Arcuri [82], and Foustalieraki, Assimakopoulos, Santamouris and Pangalou [89] found a higher $T_{S}$ of GRs than that of traditional roofs at nighttime in both hot and cold seasons. Additionally, although the maximal $\mathrm{T}_{\mathrm{s}}$ reduction in winter is not as impressive as that in summer, $\Delta \mathrm{T}_{\mathrm{S}}$ still remains positive during winter daytime. Those research outcomes are contrary to others' findings. More precisely, the $T_{\mathrm{S}}$ of a non-vegetated roof was higher than that of a GR at night-time, following Morakinyo, Dahanayake, Ng and Chow [26] and Cascone, Catania, Gagliano and Sciuto [21]. A warmer roof deck underneath the GR was observed in winter at daytime, following Boafo, Kim and Kim [81] and Cai, Feng, Yu, Xiang and Chen [85]. A combined effect of great heat storage and thermal inertia of GR components is likely to explain a warmer skin temperature of outer roof decks at night [18]. Oppositely, Cascone, Catania, Gagliano and Sciuto [21] stated that the evapotranspiration process allows GRs to release the heat accumulated during a hot summer day, which helps to maintain a lower $\mathrm{T}_{\mathrm{S}}$ of a GR at night. The negative $\Delta \mathrm{T}_{\mathrm{s}}$ at night during summer could lead to a higher cooling demand, though this is preferable to save the electricity for heating during cold seasons. On the other hand, attempts to study this topic have not yet been made. Consequently, solutions from future research are needed to avoid any unexpected impacts of GRs.

Among many attempts to study the HTC improvement, the vast majority of them analyzed the indoor dry-bulb temperature (DBT), which is also known as air temperature. On the other hand, the wet-bulb globe temperature (WBGT), which is a combined effect of air temperature and relative humidity, has received less attention. Human discomfort is primarily affected by WBGT and, hence, this variable should be involved in future works (rather than simply analyzing DBT). Moreover, the studies about whether the $\mathrm{T}_{\text {air.in }}$ improved due to the construction of GRs has been limited. Though energy savings after GR installation has been generally agreed upon, it is worthwhile to investigate the possibility of a GR-based passive-cooling system.

Another noteworthy finding is the differences in the experimental setups of measuring devices from one study to the other. It is understandable that the position of measuring devices strongly depends on the specific research aims. However, this review suggests that future research needs to apply consistent measurements for accuracy of result comparisons and performance evaluations between different studies. For example, this review detected a difference in the height of sensors for measuring the air temperature above the plant canopy. Additionally, previous studies published $\Delta \mathrm{T}_{\mathrm{s}}$ values with various positions of thermal sensors. The explanations for those chosen sensor positions are also missing. In order to properly understand the effects of GRs, an adequate number of studies with identical and appropriate experimental designs are required.

Palermo, Turco, Principato and Piro [22] and Gregoire and Clausen [43] stated that the inconsistency in published runoff reduction was due to differences in the catchment size, the length of the study period, the data-analysis approach, and the hydraulic characteristics of the GR materials. Nevertheless, no consensus about how those variables influence the GR capability of reducing runoff have been reached yet. For example, Zhang, Miao, Wang, Liu, Zhu, Zhou, Sun and Liu [49] and Razzaghmanesh and Beecham [69] highlighted the importance of an antecedent dry weather period (ADWP) in the retention capability of 
GRs. In contrast, Zhang, Szota, Fletcher, Williams and Farrell [47], Ferrans, Rey, Pérez, Rodríguez and Díaz-Granados [57], and Hakimdavar, Culligan, Finazzi, Barontini and Ranzi [51] concluded that the substrate storage capacity and initial substrate moisture content were more related to the retention performance, whereas the impact of ADWP was negligible. They also found that the selection of high water-use plants, followed by the high evapotranspiration (ET) rate, was not as important as the substrate type. In contrast, Johannessen, Muthanna and Braskerud [59] raised another debate, as they reported opposite results as the ET process made greater variations in GR performance than different GR configurations did. Kaiser et al. [114] highlighted the importance of ET by applying some solutions to increase the rate of ET. Such disputes could be resolved with extensive knowledge acquired from future works. Furthermore, Sims, Robinson, Smart and $\mathrm{O}^{\prime}$ Carroll [55] maintained that the high retention rates in some studies resulted from the inclusion of rainfall events generating no runoff $(100 \%$ retention) in the data analysis. This review recommends that all future research should apply a precipitation threshold to exclude small events with no discharge from GRs so that a proper conclusion about the runoff retention capacity of a GR system could be reached.

\section{Conclusions}

Among numerous WSUD strategies, GRs are a popularly used practice implemented to deal with the adverse impacts of climate change and to tackle various issues arising from rapid urbanization and increases in population. This paper reviewed a broad range of GR literature investigating all ecosystem services that a GR system can provide. They are HTC improvement, energy saving, runoff reduction, runoff quality enhancement, noise reduction, air quality improvement, and ecological, social, and economic benefits. This review aimed to provide readers with in-depth knowledge of GR performance during the last 10 years through the quantification of the provided benefits.

The key observations, conclusions, and recommendations that are provided on the basis of this study are as follows:

(a) Countries such as the USA and various European countries have implemented GRs quite popularly. This review also indicates that China is also taking up GRs in a big way. GRs have not been popular in developing countries due to a lack of local research about the methods for constructing GRs and their benefits. The high initial cost of construction is also a constraint in developing countries.

(b) An imbalance of GR research focuses was identified, wherein Human Thermal Comfort and runoff-related benefits were well researched when compared to other benefits. At the same time, further studies need to be undertaken on inadequately studied GR benefits, such as reduced noise and air pollution.

(c) It was found that EGR has been more commonly implemented because of numerous advantages over other types of GR. However, if only the capability of providing ecosystem services is considered, IGRs very clearly outperform EGRs. On the other hand, the intermediate type of GR, namely SIGRs, appear to have a combination of advantages taken from both EGRs and IGRs.

(d) The effectiveness of hybrid GRs was clearly observed as compared to traditional GRs. The main hybrid GRs identified in this review include photovoltaic GRs, greenblue roofs, GRs integrated with radiant cooling systems, and GRs combined with green walls. However, further studies to quantify the benefits of hybrid GRs are recommended.

(e) It is recommended that future studies are undertaken to improve upon well-known GR benefits by discovering more innovative GR construction techniques and materials. Further studies are also recommended to explore GR components that are economical as well as environmentally friendly.

(f) The impact of key influential GR parameters (e.g., substrate type and their waterholding capacity, the type of plants, and evapotranspiration rate, etc.) on its performance was continually highlighted in this review. Many studies reported contradic- 
tory outcomes on varying some of these parameters, and hence, further studies are recommended.

(g) In spite of the existence of reliable modelling tools, their application to study the largescale implementation of GRs (at a city-wide scale, catchment scale or municipality scale) has been restricted. As a result, more research and studies are necessary to transform the GR concept into one of the widespread and popularly used WSUD strategies.

(h) Recommendations to address GR limitations and obstacles in taking up GRs have been identified in this literature review.

Author Contributions: Conceptualization, N.M., M.A.U.R.T. and A.W.M.N.; methodology, C.N.N., N.M., M.A.U.R.T. and A.W.M.N.; software, C.N.N., N.M. and A.W.M.N.; validation, N.M., M.A.U.R.T. and A.W.M.N.; formal analysis, C.N.N., N.M., M.A.U.R.T. and A.W.M.N.; investigation, C.N.N.; resources, N.M. and A.W.M.N.; data curation, C.N.N.; writing-original draft preparation, C.N.N.; writing-review and editing, N.M. and C.N.N.; visualization, C.N.N.; supervision, N.M., M.A.U.R.T. and A.W.M.N.; project administration, C.N.N.; funding acquisition, N.M. and A.W.M.N. All authors have read and agreed to the published version of the manuscript.

Funding: This research received no external funding.

Institutional Review Board Statement: Not applicable.

Informed Consent Statement: Not applicable.

Conflicts of Interest: The authors declare no conflict of interest.

\section{Appendix A}

Table A1. An overview of green roof papers reviewed in this study.

\begin{tabular}{|c|c|c|c|c|c|}
\hline Number & Reference & Country & Type of GR & GR Benefit & Type of Modelling \\
\hline \multirow{2}{*}{1} & \multirow{2}{*}{$\begin{array}{c}\text { Barnhart, Pettus, Halama, McKane, } \\
\text { Mayer, Djang, Brookes and } \\
\text { Moskal [52] }\end{array}$} & \multirow{2}{*}{ USA } & EGR $^{1}$ & \multirow{2}{*}{ Runoff Reduction } & \multirow{2}{*}{ Model simulation } \\
\hline & & & $\mathrm{IGR}^{2}$ & & \\
\hline 2 & Liu et al. [115] & China & $\mathrm{SIGR}^{3}$ & $\begin{array}{l}\text { Runoff Quality } \\
\text { Improvement }\end{array}$ & Field experiment \\
\hline 3 & Feitosa and Wilkinson [35] & Australia & GR and Green Wall & HTC $^{4}$ Improvement & Field experiment \\
\hline \multirow{2}{*}{4} & \multirow{2}{*}{ He, Yu, Ozaki and Dong [18] } & \multirow{2}{*}{ China } & \multirow{2}{*}{ EGR } & HTC Improvement & \multirow{2}{*}{$\begin{array}{l}\text { Field experiment and } \\
\text { model simulation }\end{array}$} \\
\hline & & & & $\begin{array}{l}\text { Energy Use } \\
\text { Reduction }\end{array}$ & \\
\hline 5 & La Roche, Yeom and Ponce [36] & USA & $\begin{array}{l}\text { GR and Radiant } \\
\text { Cooling System }\end{array}$ & HTC Improvement & $\begin{array}{l}\text { Field experiment and } \\
\text { model simulation }\end{array}$ \\
\hline \multirow{3}{*}{6} & \multirow{3}{*}{$\begin{array}{l}\text { Ávila-Hernández, Simá, Xamán, } \\
\text { Hernández-Pérez, Téllez-Velázquez } \\
\text { and Chagolla-Aranda [19] }\end{array}$} & \multirow{3}{*}{ Mexico } & \multirow{3}{*}{ EGR } & $\begin{array}{l}\text { Ecological, Social, } \\
\text { and Economic }\end{array}$ & \multirow{3}{*}{$\begin{array}{l}\text { Field experiment and } \\
\text { model simulation }\end{array}$} \\
\hline & & & & HTC Improvement & \\
\hline & & & & $\begin{array}{l}\text { Energy Use } \\
\text { Reduction }\end{array}$ & \\
\hline 7 & Liu, Sun, Niu and Riley [20] & China & EGR & Runoff Reduction & Model simulation \\
\hline 8 & $\begin{array}{l}\text { Silva, K Najjar, WA Hammad, } \\
\text { Haddad and Vazquez [53] }\end{array}$ & Brazil & EGR & Runoff Reduction & Field experiment \\
\hline 9 & $\begin{array}{l}\text { Baek, Ligaray, Pachepsky, Chun, } \\
\text { Yoon, Park and Cho [27] }\end{array}$ & South Korea & SIGR & Runoff Reduction & $\begin{array}{l}\text { Field experiment and } \\
\text { model simulation }\end{array}$ \\
\hline \multirow{2}{*}{10} & \multirow{2}{*}{$\begin{array}{l}\text { Talebi, Bagg, Sleep and } \\
\text { O'Carroll [56] }\end{array}$} & \multirow{2}{*}{ Canada } & EGR & \multirow{2}{*}{ Runoff Reduction } & \multirow{2}{*}{ Model simulation } \\
\hline & & & SIGR & & \\
\hline
\end{tabular}


Table A1. Cont.

\begin{tabular}{|c|c|c|c|c|c|}
\hline Number & Reference & Country & Type of GR & GR Benefit & Type of Modelling \\
\hline \multirow[t]{2}{*}{11} & \multirow{2}{*}{$\begin{array}{c}\text { Gong, Yin, Li, Zhang, Wang, Fang, } \\
\text { Shi and Wang [40] }\end{array}$} & \multirow[t]{2}{*}{ China } & \multirow[t]{2}{*}{ EGR } & $\begin{array}{l}\text { Runoff Quality } \\
\text { Improvement }\end{array}$ & \multirow[t]{2}{*}{ Field experiment } \\
\hline & & & & Runoff Reduction & \\
\hline \multirow{3}{*}{12} & \multirow{3}{*}{ Cai, Feng, Yu, Xiang and Chen [85] } & \multirow{3}{*}{ China } & \multirow{3}{*}{ EGR } & HTC Improvement & \multirow{3}{*}{$\begin{array}{l}\text { Field experiment and } \\
\text { model simulation }\end{array}$} \\
\hline & & & & $\begin{array}{l}\text { Energy Use } \\
\text { Reduction }\end{array}$ & \\
\hline & & & & $\begin{array}{l}\text { Ecological, Social, } \\
\text { and Economic }\end{array}$ & \\
\hline 13 & $\begin{array}{c}\text { Jahanfar, Drake, Sleep and } \\
\text { Margolis [54] }\end{array}$ & Canada & GR and $P V^{5}$ & Runoff Reduction & Field experiment \\
\hline 14 & $\begin{array}{c}\text { Palermo, Turco, Principato and } \\
\text { Piro [22] }\end{array}$ & Italy & EGR & Runoff Reduction & $\begin{array}{l}\text { Field experiment and } \\
\text { model simulation }\end{array}$ \\
\hline 15 & $\begin{array}{l}\text { Sims, Robinson, Smart and } \\
\text { O'Carroll [55] }\end{array}$ & Canada & EGR & Runoff Reduction & $\begin{array}{l}\text { Field experiment and } \\
\text { model simulation }\end{array}$ \\
\hline \multirow[b]{2}{*}{16} & \multirow{2}{*}{ Xing, Hao, Lin, Tan and Yang [33] } & \multirow[b]{2}{*}{ China } & \multirow[b]{2}{*}{ GR and Green Wall } & HTC Improvement & \multirow{2}{*}{ Field experiment } \\
\hline & & & & $\begin{array}{l}\text { Energy Use } \\
\text { Reduction }\end{array}$ & \\
\hline 17 & Cao, Hu, Dong, Liu and Wang [83] & China & EGR & HTC Improvement & Field experiment \\
\hline 18 & $\begin{array}{l}\text { Baraldi, Neri, Costa, Facini, } \\
\text { Rapparini and Carriero [110] }\end{array}$ & Italy & IGR & $\begin{array}{l}\text { Air Quality } \\
\text { Improvement }\end{array}$ & $\mathrm{N} / \mathrm{A}$ \\
\hline \multirow[b]{2}{*}{19} & \multirow{2}{*}{ Tang and Zheng [84] } & \multirow[b]{2}{*}{ China } & \multirow[b]{2}{*}{ EGR } & HTC Improvement & \multirow[b]{2}{*}{ Field experiment } \\
\hline & & & & $\begin{array}{l}\text { Energy Use } \\
\text { Reduction }\end{array}$ & \\
\hline 20 & $\begin{array}{l}\text { Zhang, Szota, Fletcher, Williams } \\
\text { and Farrell [47] }\end{array}$ & Australia & EGR & Runoff Reduction & Indoor experiment \\
\hline \multirow{3}{*}{21} & \multirow{3}{*}{$\begin{array}{c}\text { Cascone, Catania, Gagliano and } \\
\text { Sciuto [21] }\end{array}$} & \multirow{3}{*}{ Italy } & \multirow{3}{*}{ EGR } & HTC Improvement & \multirow{3}{*}{ Model simulation } \\
\hline & & & & $\begin{array}{l}\text { Energy Use } \\
\text { Reduction }\end{array}$ & \\
\hline & & & & $\begin{array}{l}\text { Ecological, Social, } \\
\text { and Economic }\end{array}$ & \\
\hline \multirow{2}{*}{22} & \multirow{2}{*}{ Feitosa and Wilkinson [34] } & Brazil & \multirow{2}{*}{ GR and Green Wall } & \multirow{2}{*}{ HTC Improvement } & \multirow{2}{*}{ Field experiment } \\
\hline & & Australia & & & \\
\hline 23 & $\begin{array}{l}\text { Johannessen, Muthanna and } \\
\text { Braskerud [59] }\end{array}$ & Norway & EGR & Runoff Reduction & Field experiment \\
\hline 24 & Park, Kim, Dvorak and Lee [86] & South Korea & EGR & HTC Improvement & $\begin{array}{l}\text { Field experiment and } \\
\text { model simulation }\end{array}$ \\
\hline 25 & Shafique, Kim and Kyung-Ho [58] & South Korea & EGR & Runoff Reduction & Field experiment \\
\hline 26 & Berto, Stival and Rosato [106] & Italy & EGR & $\begin{array}{l}\text { Ecological, Social, } \\
\text { and Economic }\end{array}$ & Model simulation \\
\hline 27 & Lee and Jim [23] & Hong Kong & IGR & HTC Improvement & Field experiment \\
\hline 28 & $\begin{array}{l}\text { Zhang, Szota, Fletcher, Williams, } \\
\text { Werdin and Farrell [41] }\end{array}$ & Australia & EGR & Runoff Reduction & Indoor experiment \\
\hline \multirow{2}{*}{29} & \multirow{2}{*}{$\begin{array}{l}\text { Azeñas, Cuxart, Picos, Medrano, } \\
\text { Simó, López-Grifol and Gulías [87] }\end{array}$} & & & HTC Improvement & \\
\hline & & Spain & EGR & $\begin{array}{l}\text { Energy Use } \\
\text { Reduction }\end{array}$ & Field experiment \\
\hline
\end{tabular}


Table A1. Cont.

\begin{tabular}{|c|c|c|c|c|c|}
\hline Number & Reference & Country & Type of GR & GR Benefit & Type of Modelling \\
\hline \multirow{3}{*}{30} & \multirow{3}{*}{$\begin{array}{c}\text { Kratschmer, Kriechbaum and } \\
\text { Pachinger [24] }\end{array}$} & \multirow{3}{*}{ Austria } & EGR & \multirow{3}{*}{$\begin{array}{l}\text { Ecological, Social, } \\
\text { and Economic }\end{array}$} & \multirow{3}{*}{ Field experiment } \\
\hline & & & SIGR & & \\
\hline & & & IGR & & \\
\hline 31 & Rumble, Finch and Gange [107] & UK & EGR & $\begin{array}{l}\text { Ecological, Social, } \\
\text { and Economic }\end{array}$ & Field experiment \\
\hline 32 & Todorov, Driscoll and Todorova [45] & USA & EGR & Runoff Reduction & Field experiment \\
\hline \multirow[b]{2}{*}{33} & \multirow{2}{*}{$\begin{array}{c}\text { Ferrans, Rey, Pérez, Rodríguez and } \\
\text { Díaz-Granados [57] }\end{array}$} & \multirow[b]{2}{*}{ Colombia } & \multirow[b]{2}{*}{ EGR } & Runoff Reduction & \multirow[b]{2}{*}{ Field experiment } \\
\hline & & & & $\begin{array}{l}\text { Runoff Quality } \\
\text { Improvement }\end{array}$ & \\
\hline \multirow{4}{*}{34} & \multirow{4}{*}{$\begin{array}{c}\text { Morakinyo, Dahanayake, } \mathrm{Ng} \text { and } \\
\text { Chow [26] }\end{array}$} & Hong Kong & \multirow{4}{*}{ IGR } & \multirow{4}{*}{$\begin{array}{l}\text { Energy Use } \\
\text { Reduction }\end{array}$} & \multirow{4}{*}{ Model simulation } \\
\hline & & Japan & & & \\
\hline & & Egypt & & & \\
\hline & & France & & & \\
\hline \multirow[b]{2}{*}{35} & \multirow[b]{2}{*}{ He, Yu, Ozaki, Dong and Zheng [80] } & \multirow[b]{2}{*}{ China } & \multirow[b]{2}{*}{ EGR } & HTC Improvement & \multirow[b]{2}{*}{$\begin{array}{l}\text { Field experiment and } \\
\text { model simulation }\end{array}$} \\
\hline & & & & $\begin{array}{l}\text { Energy Use } \\
\text { Reduction }\end{array}$ & \\
\hline 36 & Yeom and La Roche [37] & USA & $\begin{array}{l}\text { GR and Radiant } \\
\text { Cooling System }\end{array}$ & HTC Improvement & Field experiment \\
\hline 37 & Shafique and Kim [30] & South Korea & GR and Blue Roof & HTC Improvement & Field experiment \\
\hline \multirow{2}{*}{38} & \multirow{2}{*}{$\begin{array}{l}\text { Wilkinson, Feitosa, Kaga and De } \\
\text { Franceschi [88] }\end{array}$} & Australia & \multirow{2}{*}{ GR and Green Wall } & \multirow{2}{*}{ HTC Improvement } & \multirow{2}{*}{ Field experiment } \\
\hline & & Brazil & & & \\
\hline \multirow{4}{*}{39} & \multirow{4}{*}{$\begin{array}{l}\text { Johannessen, Hanslin and } \\
\text { Muthanna [61] }\end{array}$} & Norway & \multirow{4}{*}{ EGR } & \multirow{4}{*}{ Runoff Reduction } & \multirow{4}{*}{ Model simulation } \\
\hline & & Iceland & & & \\
\hline & & Sweden & & & \\
\hline & & UK & & & \\
\hline 40 & $\begin{array}{c}\text { Bevilacqua, Mazzeo, Bruno and } \\
\text { Arcuri [82] }\end{array}$ & Italy & EGR & HTC Improvement & Field experiment \\
\hline & Foustalieraki, Assimakopoulos, & & & HTC Improvement & Field experiment and \\
\hline 41 & Santamouris and Pangalou [89] & Greece & EGR & $\begin{array}{l}\text { Energy Use } \\
\text { Reduction }\end{array}$ & model simulation \\
\hline 42 & $\begin{array}{l}\text { Soulis, Valiantzas, Ntoulas, Kargas } \\
\text { and Nektarios [60] }\end{array}$ & Greece & EGR & Runoff Reduction & $\begin{array}{l}\text { Field experiment and } \\
\text { model simulation }\end{array}$ \\
\hline & & & & HTC Improvement & \\
\hline 43 & Boafo, Kim and Kim [81] & South Korea & EGR & $\begin{array}{l}\text { Energy Use } \\
\text { Reduction }\end{array}$ & Model simulation \\
\hline 44 & Cipolla, Maglionico and Stojkov [64] & Italy & EGR & Runoff Reduction & $\begin{array}{l}\text { Field experiment and } \\
\text { model simulation }\end{array}$ \\
\hline 45 & Buffam, Mitchell and Durtsche [104] & USA & EGR & $\begin{array}{l}\text { Runoff Quality } \\
\text { Improvement }\end{array}$ & Field experiment \\
\hline & & & & $\begin{array}{l}\text { Air Quality } \\
\text { Improvement }\end{array}$ & \\
\hline 46 & $\begin{array}{l}\text { Karteris, Theodoridou, Mallinis, } \\
\text { Tsiros and Karteris [63] }\end{array}$ & Greece & SIGR & $\begin{array}{l}\text { Energy Use } \\
\text { Reduction }\end{array}$ & Model simulation \\
\hline & & & EGR & Runoff Reduction & \\
\hline
\end{tabular}


Table A1. Cont.

\begin{tabular}{|c|c|c|c|c|c|}
\hline Number & Reference & Country & Type of GR & GR Benefit & Type of Modelling \\
\hline \multirow{2}{*}{47} & \multirow{2}{*}{ El Bachawati et al. [116] } & \multirow{2}{*}{ Lebanon } & EGR & \multirow{2}{*}{$\begin{array}{l}\text { Ecological, Social, } \\
\text { and Economic }\end{array}$} & \multirow{2}{*}{ Model simulation } \\
\hline & & & IGR & & \\
\hline \multirow[b]{2}{*}{48} & \multirow{2}{*}{$\begin{array}{c}\text { Gagliano, Detommaso, Nocera and } \\
\text { Berardi [90] }\end{array}$} & \multirow[b]{2}{*}{ Italy } & \multirow[b]{2}{*}{ EGR } & HTC Improvement & \multirow[b]{2}{*}{ Model simulation } \\
\hline & & & & $\begin{array}{l}\text { Energy Use } \\
\text { Reduction }\end{array}$ & \\
\hline \multirow{2}{*}{49} & \multirow{2}{*}{ Shafique, Kim and Lee [29] } & \multirow{2}{*}{ South Korea } & \multirow{2}{*}{ GR and Blue Roof } & Runoff Reduction & \multirow{2}{*}{ Field experiment } \\
\hline & & & & HTC Improvement & \\
\hline 50 & Shafique, Lee and Kim [28] & South Korea & GR and Blue Roof & Runoff Reduction & Field experiment \\
\hline \multirow[b]{2}{*}{51} & \multirow{2}{*}{$\begin{array}{c}\text { Carpenter, Todorov, Driscoll and } \\
\text { Montesdeoca [48] }\end{array}$} & \multirow[b]{2}{*}{ USA } & \multirow[b]{2}{*}{ EGR } & Runoff Reduction & \multirow{2}{*}{ Field experiment } \\
\hline & & & & $\begin{array}{l}\text { Runoff Quality } \\
\text { Improvement }\end{array}$ & \\
\hline \multirow[b]{2}{*}{52} & \multirow[b]{2}{*}{ He, Yu, Dong and Ye [78] } & \multirow[b]{2}{*}{ China } & \multirow[b]{2}{*}{ EGR } & HTC Improvement & \multirow[b]{2}{*}{ Field experiment } \\
\hline & & & & $\begin{array}{l}\text { Energy Use } \\
\text { Reduction }\end{array}$ & \\
\hline \multirow[b]{2}{*}{53} & \multirow[b]{2}{*}{ Tam, Wang and Le [91] } & \multirow[b]{2}{*}{ Hong Kong } & EGR & HTC Improvement & \multirow[b]{2}{*}{ Field experiment } \\
\hline & & & IGR & $\begin{array}{l}\text { Ecological, Social, } \\
\text { and Economic }\end{array}$ & \\
\hline 54 & Brunetti, Šimůnek and Piro [62] & Italy & EGR & Runoff Reduction & $\begin{array}{l}\text { Field experiment and } \\
\text { model simulation }\end{array}$ \\
\hline 55 & $\begin{array}{l}\text { Nawaz, McDonald and } \\
\text { Postoyko [50] }\end{array}$ & UK & EGR & Runoff Reduction & $\begin{array}{l}\text { Field experiment and } \\
\text { model simulation }\end{array}$ \\
\hline \multirow[b]{2}{*}{56} & & & & Runoff Reduction & Field experiment and \\
\hline & Burken [68] & USA & EGR & $\begin{array}{l}\text { Runoff Quality } \\
\text { Improvement }\end{array}$ & model simulation \\
\hline 57 & Yang, Li, Sun and Ni [67] & China & EGR & Runoff Reduction & $\begin{array}{l}\text { Field experiment and } \\
\text { model simulation }\end{array}$ \\
\hline 58 & $\begin{array}{l}\text { Versini, Ramier, Berthier and De } \\
\text { Gouvello [66] }\end{array}$ & France & EGR & Runoff Reduction & $\begin{array}{l}\text { Field experiment and } \\
\text { model simulation }\end{array}$ \\
\hline & Zhang, Miao, Wang, Liu, Zhu, Zhou, & & & Runoff Reduction & \\
\hline 59 & Sun and Liu [49] & China & EGR & $\begin{array}{l}\text { Runoff Quality } \\
\text { Improvement }\end{array}$ & Field experiment \\
\hline & $\mathrm{I}, \mathrm{I}$ & & EGR & Punff Dodurtion & Field experiment \\
\hline 00 & Lee, Lee and han [00] & soutn nored & SIGR & 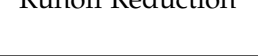 & Indoor experiment \\
\hline 61 & Vijayaraghavan and Raja [100] & India & EGR & $\begin{array}{l}\text { Runoff Quality } \\
\text { Improvement }\end{array}$ & Field experiment \\
\hline & & & EGR & Runoff reduction & \\
\hline 62 & Beecham and Razzaghmanesh [25] & Australia & IGR & $\begin{array}{l}\text { Runoff Quality } \\
\text { Improvement }\end{array}$ & Field experiment \\
\hline 63 & $\begin{array}{l}\text { Hakimdavar, Culligan, Finazzi, } \\
\text { Barontini and Ranzi [51] }\end{array}$ & USA & EGR & Runoff Reduction & $\begin{array}{l}\text { Field experiment and } \\
\text { model simulation }\end{array}$ \\
\hline 64 & Razzaghmanesh and Beecham [69] & Australia & EGR & Runoff Reduction & Field experiment \\
\hline & & & IGR & & \\
\hline 65 & Razzaghmanesh, Beecham and & Australia & EGR & Runoff Quality & Field experiment \\
\hline 0 & Kazemi [101] & Mustiand & IGR & & \\
\hline
\end{tabular}


Table A1. Cont.

\begin{tabular}{|c|c|c|c|c|c|}
\hline Number & Reference & Country & Type of GR & GR Benefit & Type of Modelling \\
\hline 66 & Vijayaraghavan and Joshi [117] & India & EGR & $\begin{array}{l}\text { Runoff Quality } \\
\text { Improvement }\end{array}$ & Field experiment \\
\hline \multirow[b]{2}{*}{67} & \multirow[b]{2}{*}{ Schweitzer and Erell [92] } & \multirow[b]{2}{*}{ Israel } & \multirow[b]{2}{*}{ EGR } & HTC Improvement & \multirow[b]{2}{*}{ Field experiment } \\
\hline & & & & $\begin{array}{l}\text { Energy Use } \\
\text { Reduction }\end{array}$ & \\
\hline 68 & Vijayaraghavan and Raja [118] & India & EGR & $\begin{array}{l}\text { Runoff Quality } \\
\text { Improvement }\end{array}$ & Field experiment \\
\hline 69 & Wong and Jim [44] & Hong Kong & EGR & Runoff Reduction & Field experiment \\
\hline 70 & Chemisana and Lamnatou [93] & Spain & GR and PV & HTC Improvement & Field experiment \\
\hline 71 & $\begin{array}{c}\text { Carson, Marasco, Culligan and } \\
\text { McGillis [10] }\end{array}$ & USA & EGR & Runoff Reduction & $\begin{array}{l}\text { Field experiment and } \\
\text { model simulation }\end{array}$ \\
\hline 72 & $\begin{array}{l}\text { Speak, Rothwell, Lindley and } \\
\text { Smith [46] }\end{array}$ & UK & SIGR & Runoff Reduction & Field experiment \\
\hline \multirow[b]{2}{*}{73} & \multirow{2}{*}{$\begin{array}{l}\text { Sun, Bou-Zeid, Wang, Zerba and } \\
\text { Ni [79] }\end{array}$} & China & \multirow[b]{2}{*}{ EGR } & HTC Improvement & \multirow{2}{*}{$\begin{array}{l}\text { Field experiment and } \\
\text { model simulation }\end{array}$} \\
\hline & & USA & & $\begin{array}{l}\text { Energy Use } \\
\text { Reduction }\end{array}$ & \\
\hline \multirow{2}{*}{74} & \multirow{2}{*}{ Connelly and Hodgson [112] } & \multirow{2}{*}{ Canada } & \multirow{2}{*}{ EGR } & \multirow{2}{*}{ Noise Reduction } & Field experiment \\
\hline & & & & & Indoor experiment \\
\hline \multirow{2}{*}{75} & \multirow{2}{*}{ Peng and Jim [3] } & \multirow{2}{*}{ Hong Kong } & EGR & \multirow{2}{*}{ HTC Improvement } & \multirow{2}{*}{$\begin{array}{l}\text { Field experiment and } \\
\text { model simulation }\end{array}$} \\
\hline & & & IGR & & \\
\hline 76 & Whittinghill, Rowe and Cregg [108] & USA & EGR & $\begin{array}{l}\text { Ecological, Social, } \\
\text { and Economic }\end{array}$ & Field experiment \\
\hline \multirow{5}{*}{77} & \multirow{5}{*}{$\begin{array}{c}\text { Ascione, Bianco, de'Rossi, Turni and } \\
\text { Vanoli [16] }\end{array}$} & Spain & & Ecological, Social, & \multirow{5}{*}{ Model simulation } \\
\hline & & UK & SIGR & and Economic & \\
\hline & & The Netherlands & & HTC Improvement & \\
\hline & & Italy & \multirow{2}{*}{ EGR } & Energy Use & \\
\hline & & Norway & & Reduction & \\
\hline \multirow{3}{*}{78} & \multirow{3}{*}{ Pandey, Hindoliya and Mod [94] } & \multirow{3}{*}{ India } & EGR & HTC Improvement & \multirow{3}{*}{ Field experiment } \\
\hline & & & SIGR & & \\
\hline & & & IGR & $\begin{array}{l}\text { Energy Use } \\
\text { Reduction }\end{array}$ & \\
\hline \multirow{2}{*}{79} & \multirow{2}{*}{$\begin{array}{l}\text { Blanusa, Monteiro, Fantozzi, Vysini, } \\
\text { Li and Cameron [95] }\end{array}$} & \multirow{2}{*}{ UK } & SIGR & HTC Improvement & Field experiment \\
\hline & & & & & Indoor experiment \\
\hline 80 & $\begin{array}{c}\text { Mickovski, Buss, McKenzie and } \\
\text { Sökmener [70] }\end{array}$ & UK & EGR & Runoff Reduction & Indoor experiment \\
\hline 81 & Qin, Wu, Chiew and Li [72] & Singapore & SIGR & HTC Improvement & Field experiment \\
\hline & & & & Runoff Reduction & \\
\hline 82 & Stovin, Vesuviano and Kasmin [42] & UK & EGR & Runoff Reduction & $\begin{array}{l}\text { Field experiment and } \\
\text { model simulation }\end{array}$ \\
\hline 83 & Nagase and Dunnett [71] & UK & EGR & Runoff Reduction & Indoor experiment \\
\hline 84 & Bianchini and Hewage [119] & Canada & EGR & Ecological, Social, & Model simulation \\
\hline & & & IGR & & \\
\hline
\end{tabular}


Table A1. Cont.

\begin{tabular}{|c|c|c|c|c|c|}
\hline Number & Reference & Country & Type of GR & GR Benefit & Type of Modelling \\
\hline \multirow[b]{2}{*}{85} & \multirow[b]{2}{*}{ Jim and Peng [96] } & \multirow[b]{2}{*}{ Hong Kong } & \multirow[b]{2}{*}{ EGR } & HTC Improvement & \multirow[b]{2}{*}{ Field experiment } \\
\hline & & & & $\begin{array}{l}\text { Energy Use } \\
\text { Reduction }\end{array}$ & \\
\hline 86 & $\begin{array}{l}\text { Vijayaraghavan, Joshi and } \\
\text { Balasubramanian [102] }\end{array}$ & Singapore & EGR & $\begin{array}{l}\text { Runoff Quality } \\
\text { Improvement }\end{array}$ & Field experiment \\
\hline \multirow[b]{2}{*}{87} & \multirow{2}{*}{$\begin{array}{l}\text { Pérez, Coma, Solé, Castell and } \\
\text { Cabeza [97] }\end{array}$} & \multirow{2}{*}{ Spain } & \multirow{2}{*}{ EGR } & HTC Improvement & \multirow{2}{*}{ Field experiment } \\
\hline & & & & $\begin{array}{l}\text { Energy Use } \\
\text { Reduction }\end{array}$ & \\
\hline 88 & Yang, Kang and Choi [113] & UK & EGR & Noise Reduction & Indoor experiment \\
\hline \multirow[b]{2}{*}{89} & \multirow[b]{2}{*}{ Gregoire and Clausen [43] } & \multirow[b]{2}{*}{ USA } & \multirow[b]{2}{*}{ EGR } & Runoff Reduction & \multirow[b]{2}{*}{$\begin{array}{l}\text { Field experiment and } \\
\text { model simulation }\end{array}$} \\
\hline & & & & $\begin{array}{l}\text { Runoff Quality } \\
\text { Improvement }\end{array}$ & \\
\hline 90 & MacIvor and Lundholm [38] & Canada & IGR & $\begin{array}{l}\text { Ecological, Social, } \\
\text { and Economic }\end{array}$ & Field experiment \\
\hline 91 & Tsang and Jim [120] & Hong Kong & EGR & $\begin{array}{l}\text { Ecological, Social, } \\
\text { and Economic }\end{array}$ & Model simulation \\
\hline 92 & Alsup et al. [121] & USA & EGR & $\begin{array}{l}\text { Runoff quality } \\
\text { Improvement }\end{array}$ & Field experiment \\
\hline \multirow[t]{2}{*}{93} & \multirow[t]{2}{*}{ Beck, Johnson and Spolek [74] } & \multirow[t]{2}{*}{ USA } & \multirow[t]{2}{*}{ EGR } & $\begin{array}{l}\text { Runoff Quality } \\
\text { Improvement }\end{array}$ & \multirow[t]{2}{*}{ Indoor experiment } \\
\hline & & & & Runoff Reduction & \\
\hline \multirow[t]{2}{*}{94} & \multirow[t]{2}{*}{ Buccola and Spolek [73] } & \multirow[t]{2}{*}{ USA } & \multirow[t]{2}{*}{ EGR } & $\begin{array}{l}\text { Runoff Quality } \\
\text { Improvement }\end{array}$ & \multirow[t]{2}{*}{ Indoor experiment } \\
\hline & & & & Runoff Reduction & \\
\hline \multirow[b]{2}{*}{95} & \multirow{2}{*}{$\begin{array}{l}\text { Getter, Rowe, Andresen and } \\
\text { Wichman [98] }\end{array}$} & \multirow[b]{2}{*}{ USA } & \multirow[b]{2}{*}{ EGR } & HTC Improvement & \multirow{2}{*}{ Field experiment } \\
\hline & & & & $\begin{array}{l}\text { Energy Use } \\
\text { Reduction }\end{array}$ & \\
\hline \multirow[b]{2}{*}{96} & \multirow{2}{*}{ Hui and Chan [99] } & & & HTC Improvement & Field experiment and \\
\hline & & Hong Kong & GR and PV & $\begin{array}{l}\text { Energy Use } \\
\text { Reduction }\end{array}$ & model simulation \\
\hline 97 & Palla Gnecco and Lanza [77] & Italy & SIGR & Runoff Reduction & Field experiment \\
\hline & & & EGR & & Indoor experiment \\
\hline 98 & Voyde, Fassman and Simcock [76] & New Zealand & EGR & Runoff Reduction & Field experiment \\
\hline 99 & Roehr and Kong [75] & Canada & $\mathrm{FCR}$ & Runeff Reduction & Modolsimulation \\
\hline צת & & China & LUI & Numon Necuctiont & viouer simturation \\
\hline 100 & $\begin{array}{l}\text { Li, Wai, Li, Zhan, Ho, Li and } \\
\text { Lam [111] }\end{array}$ & Hong Kong & IGR & $\begin{array}{l}\text { Air Quality } \\
\text { Improvement }\end{array}$ & $\begin{array}{l}\text { Indoor experiment, } \\
\text { Field experiment, } \\
\text { and model } \\
\text { simulation }\end{array}$ \\
\hline 101 & Alsup, Ebbs and Retzlaff [103] & USA & EGR & $\begin{array}{l}\text { Runoff Quality } \\
\text { Improvement }\end{array}$ & Indoor experiment \\
\hline 102 & Niu et al. [122] & USA & EGR & $\begin{array}{l}\text { Ecological, Social, } \\
\text { and Economic }\end{array}$ & Model simulation \\
\hline
\end{tabular}

${ }^{1}$ Extensive Green Roof, ${ }^{2}$ Intensive Green Roof, ${ }^{3}$ Semi-intensive Green Roof, ${ }^{4}$ Human Thermal Comfort,

${ }^{5}$ Photovoltaic. 


\section{References}

1. Shafique, M.; Kim, R.; Rafiq, M. Green roof benefits, opportunities and challenges-A review. Renew. Sustain. Energy Rev. 2018, 90, 757-773. [CrossRef]

2. Vijayaraghavan, K. Green roofs: A critical review on the role of components, benefits, limitations and trends. Renew. Sustain. Energy Rev. 2016, 57, 740-752. [CrossRef]

3. Peng, L.L.; Jim, C.Y. Green-roof effects on neighborhood microclimate and human thermal sensation. Energies 2013, 6, 598-618. [CrossRef]

4. Saadatian, O.; Sopian, K.; Salleh, E.; Lim, C.; Riffat, S.; Saadatian, E.; Toudeshki, A.; Sulaiman, M. A review of energy aspects of green roofs. Renew. Sustain. Energy Rev. 2013, 23, 155-168. [CrossRef]

5. Akther, M.; He, J.; Chu, A.; Huang, J.; Van Duin, B. A review of green roof applications for managing urban stormwater in different climatic zones. Sustainability 2018, 10, 2864. [CrossRef]

6. Van Mechelen, C.; Dutoit, T.; Hermy, M. Adapting green roof irrigation practices for a sustainable future: A review. Sustain. Cities Soc. 2015, 19, 74-90. [CrossRef]

7. Vacek, P.; Struhala, K.; Matějka, L. Life-cycle study on semi intensive green roofs. J. Clean. Prod. 2017, 154, 203-213. [CrossRef]

8. Blank, L.; Vasl, A.; Levy, S.; Grant, G.; Kadas, G.; Dafni, A.; Blaustein, L. Directions in green roof research: A bibliometric study. Build. Environ. 2013, 66, 23-28. [CrossRef]

9. Zheng, X.; Zou, Y.; Lounsbury, A.W.; Wang, C.; Wang, R.J.R. Green roofs for stormwater runoff retention: A global quantitative synthesis of the performance. Resour. Conserv. Recycl. 2021, 170, 105577. [CrossRef]

10. Carson, T.; Marasco, D.; Culligan, P.; McGillis, W. Hydrological performance of extensive green roofs in New York City: Observations and multi-year modeling of three full-scale systems. Environ. Res. Lett. 2013, 8, 024036. [CrossRef]

11. Francis, L.F.M.; Jensen, M.B. Benefits of green roofs: A systematic review of the evidence for three ecosystem services. Urban For. Urban Green. 2017, 28, 167-176. [CrossRef]

12. Berardi, U.; GhaffarianHoseini, A.; GhaffarianHoseini, A. State-of-the-art analysis of the environmental benefits of green roofs. Appl. Energy 2014, 115, 411-428. [CrossRef]

13. Berndtsson, J.C. Green roof performance towards management of runoff water quantity and quality: A review. Ecol. Eng. 2010, 36, 351-360. [CrossRef]

14. Hashemi, S.S.G.; Mahmud, H.B.; Ashraf, M.A. Performance of green roofs with respect to water quality and reduction of energy consumption in tropics: A review. Renew. Sustain. Energy Rev. 2015, 52, 669-679. [CrossRef]

15. Chen, C.-F. Performance evaluation and development strategies for green roofs in Taiwan: A review. Ecol. Eng. 2013, 52, 51-58. [CrossRef]

16. Ascione, F.; Bianco, N.; de'Rossi, F.; Turni, G.; Vanoli, G.P. Green roofs in European climates. Are effective solutions for the energy savings in air-conditioning? Appl. Energy 2013, 104, 845-859. [CrossRef]

17. Köhler, M.; Kaiser, D.J.B. Evidence of the climate mitigation effect of green roofs-A 20-year weather study on an Extensive Green Roof (EGR) in Northeast Germany. 2019, 9, 157. Buildings 2019, 9, 157. [CrossRef]

18. He, Y.; Yu, H.; Ozaki, A.; Dong, N. Thermal and energy performance of green roof and cool roof: A comparison study in Shanghai area. J. Clean. Prod. 2020, 267, 122205. [CrossRef]

19. Ávila-Hernández, A.; Simá, E.; Xamán, J.; Hernández-Pérez, I.; Téllez-Velázquez, E.; Chagolla-Aranda, M. Test box experiment and simulations of a green-roof: Thermal and energy performance of a residential building standard for Mexico. Energy Build. 2020, 209, 109709. [CrossRef]

20. Liu, L.; Sun, L.; Niu, J.; Riley, W.J. Modeling green roof potential to mitigate urban flooding in a Chinese city. Water 2020, $12,2082$. [CrossRef]

21. Cascone, S.; Catania, F.; Gagliano, A.; Sciuto, G. A comprehensive study on green roof performance for retrofitting existing buildings. Build. Environ. 2018, 136, 227-239. [CrossRef]

22. Palermo, S.A.; Turco, M.; Principato, F.; Piro, P. Hydrological effectiveness of an extensive green roof in Mediterranean climate. Water 2019, 11, 1378. [CrossRef]

23. Lee, L.S.; Jim, C.Y. Thermal-cooling performance of subtropical green roof with deep substrate and woodland vegetation. Ecol. Eng. 2018, 119, 8-18. [CrossRef]

24. Kratschmer, S.; Kriechbaum, M.; Pachinger, B. Buzzing on top: Linking wild bee diversity, abundance and traits with green roof qualities. Urban Ecosyst. 2018, 21, 429-446. [CrossRef]

25. Beecham, S.; Razzaghmanesh, M. Water quality and quantity investigation of green roofs in a dry climate. Water Res. 2015, 70, 370-384. [CrossRef]

26. Morakinyo, T.E.; Dahanayake, K.K.C.; Ng, E.; Chow, C.L. Temperature and cooling demand reduction by green-roof types in different climates and urban densities: A co-simulation parametric study. Energy Build. 2017, 145, 226-237. [CrossRef]

27. Baek, S.; Ligaray, M.; Pachepsky, Y.; Chun, J.A.; Yoon, K.-S.; Park, Y.; Cho, K.H. Assessment of a green roof practice using the coupled SWMM and HYDRUS models. J. Environ. Manag. 2020, 261, 109920. [CrossRef]

28. Shafique, M.; Lee, D.; Kim, R. A field study to evaluate runoff quantity from blue roof and green blue roof in an urban area. Int. J. Control Autom. 2016, 9, 59-68. [CrossRef]

29. Shafique, M.; Kim, R.; Lee, D. The potential of green-blue roof to manage storm water in urban areas. Nat. Environ. Pollut. Technol. 2016, 15, 715 . 
30. Shafique, M.; Kim, R. Application of green blue roof to mitigate heat island phenomena and resilient to climate change in urban areas: A case study from Seoul, Korea. J. Water Land Dev. 2017, 33, 165-170. [CrossRef]

31. Lamnatou, C.; Chemisana, D. A critical analysis of factors affecting photovoltaic-green roof performance. Renew. Sustain. Energy Rev. 2015, 43, 264-280. [CrossRef]

32. Kaiser, A.; Zamora, B.; Mazón, R.; García, J.; Vera, F. Experimental study of cooling BIPV modules by forced convection in the air channel. Appl. Energy 2014, 135, 88-97. [CrossRef]

33. Xing, Q.; Hao, X.; Lin, Y.; Tan, H.; Yang, K. Experimental investigation on the thermal performance of a vertical greening system with green roof in wet and cold climates during winter. Energy Build. 2019, 183, 105-117. [CrossRef]

34. Feitosa, R.C.; Wilkinson, S.J. Attenuating heat stress through green roof and green wall retrofit. Build. Environ. 2018, 140, 11-22. [CrossRef]

35. Feitosa, R.C.; Wilkinson, S.J. Small-scale experiments of seasonal heat stress attenuation through a combination of green roof and green walls. J. Clean. Prod. 2020, 250, 119443. [CrossRef]

36. La Roche, P.; Yeom, D.J.; Ponce, A. Passive cooling with a hybrid green roof for extreme climates. Energy Build. 2020, $224,110243$. [CrossRef]

37. Yeom, D.; La Roche, P. Investigation on the cooling performance of a green roof with a radiant cooling system. Energy Build. 2017, 149, 26-37. [CrossRef]

38. MacIvor, J.S.; Lundholm, J. Insect species composition and diversity on intensive green roofs and adjacent level-ground habitats. Urban Ecosyst. 2011, 14, 225-241. [CrossRef]

39. Francis, R.A.; Lorimer, J. Urban reconciliation ecology: The potential of living roofs and walls. J. Environ. Manag. 2011, 92, 1429-1437. [CrossRef]

40. Gong, Y.; Yin, D.; Li, J.; Zhang, X.; Wang, W.; Fang, X.; Shi, H.; Wang, Q. Performance assessment of extensive green roof runoff flow and quality control capacity based on pilot experiments. Sci. Total Environ. 2019, 687, 505-515. [CrossRef]

41. Zhang, Z.; Szota, C.; Fletcher, T.D.; Williams, N.S.; Werdin, J.; Farrell, C. Influence of plant composition and water use strategies on green roof stormwater retention. Sci. Total Environ. 2018, 625, 775-781. [CrossRef]

42. Stovin, V.; Vesuviano, G.; Kasmin, H. The hydrological performance of a green roof test bed under UK climatic conditions. J. Hydrol. 2012, 414, 148-161. [CrossRef]

43. Gregoire, B.G.; Clausen, J.C. Effect of a modular extensive green roof on stormwater runoff and water quality. Ecol. Eng. 2011, 37, 963-969. [CrossRef]

44. Wong, G.K.; Jim, C.Y. Quantitative hydrologic performance of extensive green roof under humid-tropical rainfall regime. Ecol. Eng. 2014, 70, 366-378. [CrossRef]

45. Todorov, D.; Driscoll, C.T.; Todorova, S. Long-term and seasonal hydrologic performance of an extensive green roof. Hydrol. Processes 2018, 32, 2471-2482. [CrossRef]

46. Speak, A.; Rothwell, J.; Lindley, S.; Smith, C. Rainwater runoff retention on an aged intensive green roof. Sci. Total Environ. 2013, 461, 28-38. [CrossRef]

47. Zhang, Z.; Szota, C.; Fletcher, T.D.; Williams, N.S.; Farrell, C. Green roof storage capacity can be more important than evapotranspiration for retention performance. J. Environ. Manag. 2019, 232, 404-412. [CrossRef] [PubMed]

48. Carpenter, C.M.; Todorov, D.; Driscoll, C.T.; Montesdeoca, M. Water quantity and quality response of a green roof to storm events: Experimental and monitoring observations. Environ. Pollut. 2016, 218, 664-672. [CrossRef] [PubMed]

49. Zhang, Q.; Miao, L.; Wang, X.; Liu, D.; Zhu, L.; Zhou, B.; Sun, J.; Liu, J. The capacity of greening roof to reduce stormwater runoff and pollution. Landsc. Urban Plan. 2015, 144, 142-150. [CrossRef]

50. Nawaz, R.; McDonald, A.; Postoyko, S. Hydrological performance of a full-scale extensive green roof located in a temperate climate. Ecol. Eng. 2015, 82, 66-80. [CrossRef]

51. Hakimdavar, R.; Culligan, P.J.; Finazzi, M.; Barontini, S.; Ranzi, R. Scale dynamics of extensive green roofs: Quantifying the effect of drainage area and rainfall characteristics on observed and modeled green roof hydrologic performance. Ecol. Eng. 2014, 73, 494-508. [CrossRef]

52. Barnhart, B.; Pettus, P.; Halama, J.; McKane, R.; Mayer, P.; Djang, K.; Brookes, A.; Moskal, L.M. Modeling the hydrologic effects of watershed-scale green roof implementation in the Pacific Northwest, United States. J. Environ. Manag. 2021, $277,111418$. [CrossRef]

53. Silva, M.d.; K Najjar, M.; WA Hammad, A.; Haddad, A.; Vazquez, E. Assessing the retention capacity of an experimental green roof prototype. Water 2020, 12, 90. [CrossRef]

54. Jahanfar, A.; Drake, J.; Sleep, B.; Margolis, L. Evaluating the shading effect of photovoltaic panels on green roof discharge reduction and plant growth. J. Hydrol. 2019, 568, 919-928. [CrossRef]

55. Sims, A.W.; Robinson, C.E.; Smart, C.C.; O'Carroll, D.M. Mechanisms controlling green roof peak flow rate attenuation. J. Hydrol. 2019, 577, 123972. [CrossRef]

56. Talebi, A.; Bagg, S.; Sleep, B.E.; O'Carroll, D.M. Water retention performance of green roof technology: A comparison of canadian climates. Ecol. Eng. 2019, 126, 1-15. [CrossRef]

57. Ferrans, P.; Rey, C.V.; Pérez, G.; Rodríguez, J.P.; Díaz-Granados, M. Effect of green roof configuration and hydrological variables on runoff water quantity and quality. Water 2018, 10, 960. [CrossRef] 
58. Shafique, M.; Kim, R.; Kyung-Ho, K. Green roof for stormwater management in a highly urbanized area: The case of Seoul, Korea. Sustainability 2018, 10, 584. [CrossRef]

59. Johannessen, B.G.; Muthanna, T.M.; Braskerud, B.C. Detention and retention behavior of four extensive green roofs in three nordic climate zones. Water 2018, 10, 671. [CrossRef]

60. Soulis, K.X.; Valiantzas, J.D.; Ntoulas, N.; Kargas, G.; Nektarios, P.A. Simulation of green roof runoff under different substrate depths and vegetation covers by coupling a simple conceptual and a physically based hydrological model. J. Environ. Manag. 2017, 200, 434-445. [CrossRef] [PubMed]

61. Johannessen, B.G.; Hanslin, H.M.; Muthanna, T.M. Green roof performance potential in cold and wet regions. Ecol. Eng. 2017, 106, 436-447. [CrossRef]

62. Brunetti, G.; Šimůnek, J.; Piro, P. A comprehensive analysis of the variably saturated hydraulic behavior of a green roof in a mediterranean climate. Vadose Zone J. 2016, 15, 1-17. [CrossRef]

63. Karteris, M.; Theodoridou, I.; Mallinis, G.; Tsiros, E.; Karteris, A. Towards a green sustainable strategy for Mediterranean cities: Assessing the benefits of large-scale green roofs implementation in Thessaloniki, Northern Greece, using environmental modelling, GIS and very high spatial resolution remote sensing data. Renew. Sustain. Energy Rev. 2016, 58, 510-525. [CrossRef]

64. Cipolla, S.S.; Maglionico, M.; Stojkov, I. A long-term hydrological modelling of an extensive green roof by means of SWMM. Ecol. Eng. 2016, 95, 876-887. [CrossRef]

65. Lee, J.Y.; Lee, M.J.; Han, M. A pilot study to evaluate runoff quantity from green roofs. J. Environ. Manag. 2015, 152, 171-176. [CrossRef]

66. Versini, P.-A.; Ramier, D.; Berthier, E.; De Gouvello, B. Assessment of the hydrological impacts of green roof: From building scale to basin scale. J. Hydrol. 2015, 524, 562-575. [CrossRef]

67. Yang, W.-Y.; Li, D.; Sun, T.; Ni, G.-H. Saturation-excess and infiltration-excess runoff on green roofs. Ecol. Eng. 2015, 74, 327-336. [CrossRef]

68. Harper, G.E.; Limmer, M.A.; Showalter, W.E.; Burken, J.G. Nine-month evaluation of runoff quality and quantity from an experiential green roof in Missouri, USA. Ecol. Eng. 2015, 78, 127-133. [CrossRef]

69. Razzaghmanesh, M.; Beecham, S. The hydrological behaviour of extensive and intensive green roofs in a dry climate. Sci. Total Environ. 2014, 499, 284-296. [CrossRef]

70. Mickovski, S.B.; Buss, K.; McKenzie, B.M.; Sökmener, B. Laboratory study on the potential use of recycled inert construction waste material in the substrate mix for extensive green roofs. Ecol. Eng. 2013, 61, 706-714. [CrossRef]

71. Nagase, A.; Dunnett, N. Amount of water runoff from different vegetation types on extensive green roofs: Effects of plant species, diversity and plant structure. Landsc. Urban Plan. 2012, 104, 356-363. [CrossRef]

72. Qin, X.; Wu, X.; Chiew, Y.; Li, Y. A green roof test bed for stormwater management and reduction of urban heat island effect in Singapore. Br. J. Environ. Clim. Chang. 2012, 2, 410-420. [CrossRef]

73. Buccola, N.; Spolek, G. A pilot-scale evaluation of greenroof runoff retention, detention, and quality. Water Air Soil Pollut. 2011, 216, 83-92. [CrossRef]

74. Beck, D.A.; Johnson, G.R.; Spolek, G.A. Amending greenroof soil with biochar to affect runoff water quantity and quality. Environ. Pollut. 2011, 159, 2111-2118. [CrossRef] [PubMed]

75. Roehr, D.; Kong, Y. Runoff reduction effects of green roofs in Vancouver, BC, Kelowna, BC, and Shanghai, PR China. Can. Water Resour. J. 2010, 35, 53-68. [CrossRef]

76. Voyde, E.; Fassman, E.; Simcock, R. Hydrology of an extensive living roof under sub-tropical climate conditions in Auckland, New Zealand. J. Hydrol. 2010, 394, 384-395. [CrossRef]

77. Palla, A.; Gnecco, I.; Lanza, L.G. Hydrologic restoration in the urban environment using green roofs. Water 2010, 2, 140-154. [CrossRef]

78. He, Y.; Yu, H.; Dong, N.; Ye, H. Thermal and energy performance assessment of extensive green roof in summer: A case study of a lightweight building in Shanghai. Energy Build. 2016, 127, 762-773. [CrossRef]

79. Sun, T.; Bou-Zeid, E.; Wang, Z.-H.; Zerba, E.; Ni, G.-H. Hydrometeorological determinants of green roof performance via a vertically-resolved model for heat and water transport. Build. Environ. 2013, 60, 211-224. [CrossRef]

80. He, Y.; Yu, H.; Ozaki, A.; Dong, N.; Zheng, S. Long-term thermal performance evaluation of green roof system based on two new indexes: A case study in Shanghai area. Build. Environ. 2017, 120, 13-28. [CrossRef]

81. Boafo, F.E.; Kim, J.-T.; Kim, J.-H. Evaluating the impact of green roof evapotranspiration on annual building energy performance. Int. J. Green Energy 2017, 14, 479-489. [CrossRef]

82. Bevilacqua, P.; Mazzeo, D.; Bruno, R.; Arcuri, N. Surface temperature analysis of an extensive green roof for the mitigation of urban heat island in southern mediterranean climate. Energy Build. 2017, 150, 318-327. [CrossRef]

83. Cao, J.; Hu, S.; Dong, Q.; Liu, L.; Wang, Z. Green roof cooling contributed by plant species with different photosynthetic strategies. Energy Build. 2019, 195, 45-50. [CrossRef]

84. Tang, M.; Zheng, X. Experimental study of the thermal performance of an extensive green roof on sunny summer days. Appl. Energy 2019, 242, 1010-1021. [CrossRef]

85. Cai, L.; Feng, X.-P.; Yu, J.-Y.; Xiang, Q.-C.; Chen, R. Reduction in carbon dioxide emission and energy savings obtained by using a green roof. Aerosol Air Qual. Res. 2019, 19, 2432-2445. [CrossRef] 
86. Park, J.; Kim, J.-H.; Dvorak, B.; Lee, D.K. The role of green roofs on microclimate mitigation effect to local climates in summer. Int. J. Environ. Res. 2018, 12, 671-679. [CrossRef]

87. Azeñas, V.; Cuxart, J.; Picos, R.; Medrano, H.; Simó, G.; López-Grifol, A.; Gulías, J. Thermal regulation capacity of a green roof system in the mediterranean region: The effects of vegetation and irrigation level. Energy Build. 2018, 164, 226-238. [CrossRef]

88. Wilkinson, S.; Feitosa, R.C.; Kaga, I.T.; De Franceschi, I.H. Evaluating the thermal performance of retrofitted lightweight green roofs and walls in Sydney and Rio de Janeiro. Procedia Eng. 2017, 180, 231-240. [CrossRef]

89. Foustalieraki, M.; Assimakopoulos, M.; Santamouris, M.; Pangalou, H. Energy performance of a medium scale green roof system installed on a commercial building using numerical and experimental data recorded during the cold period of the year. Energy Build. 2017, 135, 33-38. [CrossRef]

90. Gagliano, A.; Detommaso, M.; Nocera, F.; Berardi, U. The adoption of green roofs for the retrofitting of existing buildings in the Mediterranean climate. Int. J. Sustain. Build. Technol. Urban Dev. 2016, 7, 116-129. [CrossRef]

91. Tam, V.W.; Wang, J.; Le, K.N. Thermal insulation and cost effectiveness of green-roof systems: An empirical study in Hong Kong. Build. Environ. 2016, 110, 46-54. [CrossRef]

92. Schweitzer, O.; Erell, E. Evaluation of the energy performance and irrigation requirements of extensive green roofs in a waterscarce Mediterranean climate. Energy Build. 2014, 68, 25-32. [CrossRef]

93. Chemisana, D.; Lamnatou, C. Photovoltaic-green roofs: An experimental evaluation of system performance. Appl. Energy 2014, 119, 246-256. [CrossRef]

94. Pandey, S.; Hindoliya, D.; Mod, R. Experimental investigation on green roofs over buildings. Int. J. Low-Carbon Technol. 2013, 8 , 37-42. [CrossRef]

95. Blanusa, T.; Monteiro, M.M.V.; Fantozzi, F.; Vysini, E.; Li, Y.; Cameron, R.W. Alternatives to Sedum on green roofs: Can broad leaf perennial plants offer better 'cooling service'? Build. Environ. 2013, 59, 99-106. [CrossRef]

96. Jim, C.Y.; Peng, L.L. Weather effect on thermal and energy performance of an extensive tropical green roof. Urban For. Urban Green. 2012, 11, 73-85. [CrossRef]

97. Pérez, G.; Coma, J.; Solé, C.; Castell, A.; Cabeza, L.F. Green roofs as passive system for energy savings when using rubber crumbs as drainage layer. Energy Procedia 2012, 30, 452-460. [CrossRef]

98. Getter, K.L.; Rowe, D.B.; Andresen, J.A.; Wichman, I.S. Seasonal heat flux properties of an extensive green roof in a Midwestern US climate. Energy Build. 2011, 43, 3548-3557. [CrossRef]

99. Hui, S.C.; Chan, S.-C. Integration of green roof and solar photovoltaic systems. In Proceedings of the Joint Symposium 2011: Integrated Building Design in the New Era of Sustainability, Kowloon Shangri-la Hotel, Tsim Sha Tsui East, Kowloon, Hong Kong, 22 November 2011; pp. 1-12.

100. Vijayaraghavan, K.; Raja, F.D. Pilot-scale evaluation of green roofs with Sargassum biomass as an additive to improve runoff quality. Ecol. Eng. 2015, 75, 70-78. [CrossRef]

101. Razzaghmanesh, M.; Beecham, S.; Kazemi, F. Impact of green roofs on stormwater quality in a South Australian urban environment. Sci. Total Environ. 2014, 470, 651-659. [CrossRef]

102. Vijayaraghavan, K.; Joshi, U.; Balasubramanian, R. A field study to evaluate runoff quality from green roofs. Water Res. 2012, 46, 1337-1345. [CrossRef]

103. Alsup, S.; Ebbs, S.; Retzlaff, W. The exchangeability and leachability of metals from select green roof growth substrates. Urban Ecosyst. 2010, 13, 91-111. [CrossRef]

104. Buffam, I.; Mitchell, M.E.; Durtsche, R.D. Environmental drivers of seasonal variation in green roof runoff water quality. Ecol. Eng. 2016, 91, 506-514. [CrossRef]

105. Bianchini, F.; Hewage, K. How "green" are the green roofs? Lifecycle analysis of green roof materials. Build. Environ. 2012, 48, 57-65. [CrossRef]

106. Berto, R.; Stival, C.A.; Rosato, P. Enhancing the environmental performance of industrial settlements: An economic evaluation of extensive green roof competitiveness. Build. Environ. 2018, 127, 58-68. [CrossRef]

107. Rumble, H.; Finch, P.; Gange, A.C. Green roof soil organisms: Anthropogenic assemblages or natural communities? Appl. Soil Ecol. 2018, 126, 11-20. [CrossRef]

108. Whittinghill, L.J.; Rowe, D.B.; Cregg, B.M. Evaluation of vegetable production on extensive green roofs. Agroecol. Sustain. Food Syst. 2013, 37, 465-484. [CrossRef]

109. Yang, J.; Yu, Q.; Gong, P. Quantifying air pollution removal by green roofs in Chicago. Atmos. Environ. 2008, 42, 7266-7273. [CrossRef]

110. Baraldi, R.; Neri, L.; Costa, F.; Facini, O.; Rapparini, F.; Carriero, G. Ecophysiological and micromorphological characterization of green roof vegetation for urban mitigation. Urban For. Urban Green. 2019, 37, 24-32. [CrossRef]

111. Li, J.-f.; Wai, O.W.; Li, Y.S.; Zhan, J.-m.; Ho, Y.A.; Li, J.; Lam, E. Effect of green roof on ambient $\mathrm{CO}_{2}$ concentration. Build. Environ. 2010, 45, 2644-2651. [CrossRef]

112. Connelly, M.; Hodgson, M. Experimental investigation of the sound transmission of vegetated roofs. Appl. Acoust. 2013, 74, 1136-1143. [CrossRef]

113. Yang, H.S.; Kang, J.; Choi, M.S. Acoustic effects of green roof systems on a low-profiled structure at street level. Build. Environ. 2012, 50, 44-55. [CrossRef] 
114. Kaiser, D.; Köhler, M.; Schmidt, M.; Wolff, F.J.B. Increasing evapotranspiration on extensive green roofs by changing substrate depths, construction, and additional irrigation. Buildings 2019, 9, 173. [CrossRef]

115. Liu, L.; Cao, J.; Ali, M.; Zhang, J.; Wang, Z. Impact of green roof plant species on domestic wastewater treatment. Environ. Adv. 2021, 4, 100059. [CrossRef]

116. El Bachawati, M.; Manneh, R.; Belarbi, R.; Dandres, T.; Nassab, C.; El Zakhem, H. Cradle-to-gate Life Cycle Assessment of traditional gravel ballasted, white reflective, and vegetative roofs: A Lebanese case study. J. Clean. Prod. 2016, 137, 833-842. [CrossRef]

117. Vijayaraghavan, K.; Joshi, U.M. Can green roof act as a sink for contaminants? A methodological study to evaluate runoff quality from green roofs. Environ. Pollut. 2014, 194, 121-129. [CrossRef] [PubMed]

118. Vijayaraghavan, K.; Raja, F.D. Design and development of green roof substrate to improve runoff water quality: Plant growth experiments and adsorption. Water Res. 2014, 63, 94-101. [CrossRef]

119. Bianchini, F.; Hewage, K. Probabilistic social cost-benefit analysis for green roofs: A lifecycle approach. Build. Environ. 2012, 58, 152-162. [CrossRef]

120. Tsang, S.; Jim, C.Y. Game-theory approach for resident coalitions to allocate green-roof benefits. Environ. Plan. A 2011, 43, 363-377. [CrossRef]

121. Alsup, S.E.; Ebbs, S.D.; Battaglia, L.L.; Retzlaff, W.A. Heavy metals in leachate from simulated green roof systems. Ecol. Eng. 2011, 37, 1709-1717. [CrossRef]

122. Niu, H.; Clark, C.; Zhou, J.; Adriaens, P. Scaling of economic benefits from green roof implementation in Washington, DC. Environ. Sci. Technol. 2010, 44, 4302-4308. [CrossRef] [PubMed] 